Peter Mausbach, Robin Fingerhut, Jadran Vrabec

\title{
Structure and dynamics of the Lennard- Jones fcc-solid focusing on melting precursors
}

Journal article | Accepted manuscript (Postprint)

This version is available at https://doi.org/10.14279/depositonce-10831

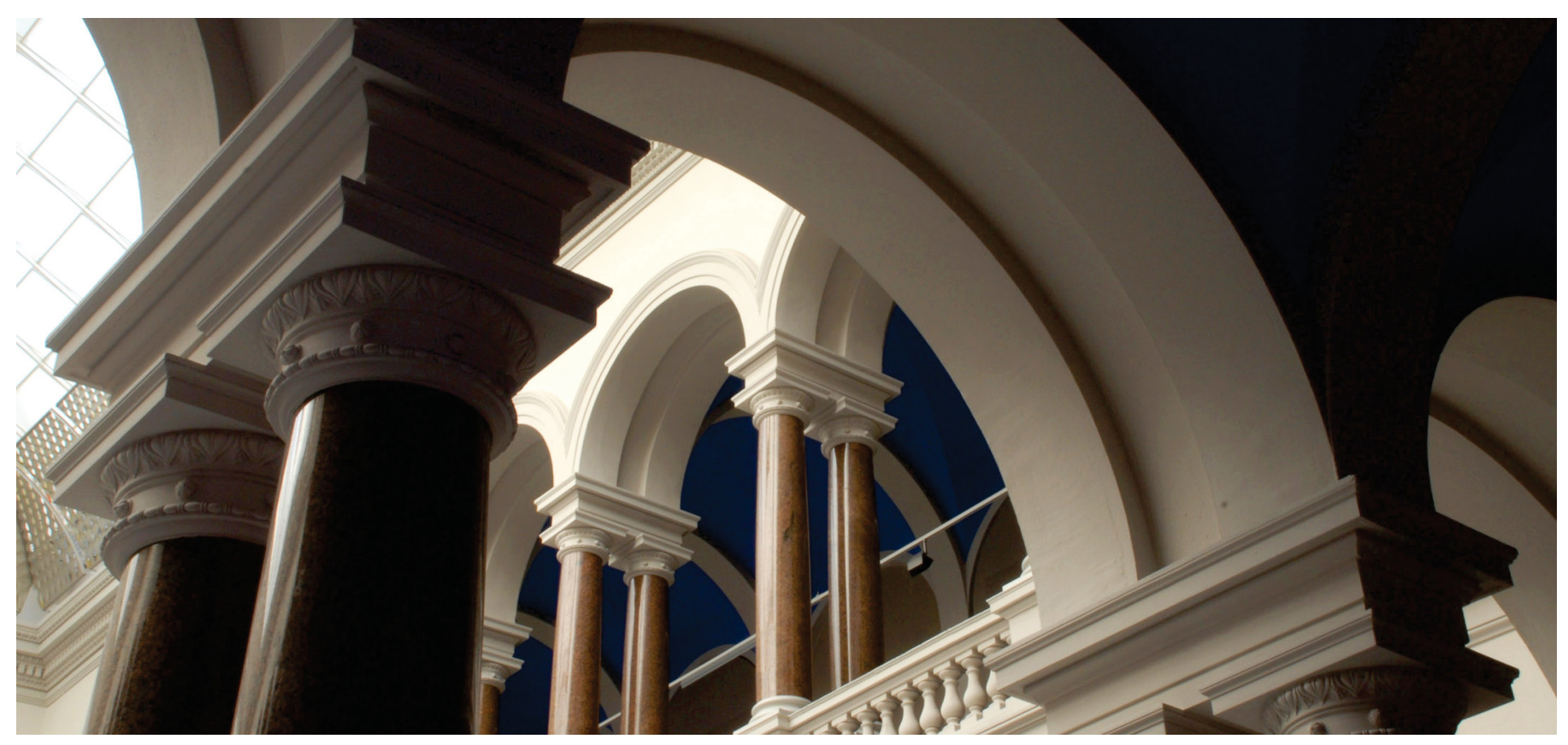

This article may be downloaded for personal use only. Any other use requires prior permission of the author and AIP Publishing. This article appeared in J. Chem. Phys. 153, 104506 (2020) and may be found at https://doi.org/10.1063/5.0015371.

Mausbach, P., Fingerhut, R., \& Vrabec, J. (2020). Structure and dynamics of the Lennard-Jones fcc-solid focusing on melting precursors. The Journal of Chemical Physics, 153(10), 104506.

https://doi.org/10.1063/5.0015371 


\title{
Structure and dynamics of the Lennard-Jones fcc-solid focusing on melting precursors
}

\author{
Peter Mausbach $^{\mathrm{a}}$, Robin Fingerhut ${ }^{\mathrm{b}}$, and Jadran Vrabec ${ }^{\mathrm{b}}{ }^{*}$ \\ ${ }^{a}$ Plant and Process Engineering, Technical University of Cologne, 50678 Cologne, Germany \\ ${ }^{\mathrm{b}}$ Thermodynamics and Process Engineering, Technical University Berlin, 10587 Berlin, \\ Germany \\ ${ }^{*}$ Corresponding author, E-mail address: vrabec@tu-berlin.de
}

\begin{abstract}
The Lennard-Jones potential is taken as a basis to study structure and dynamics of the face centered cubic (fcc) solid along an isochore from low temperatures up to the solid/fluid transition. The $\mathrm{Z}$ method is applied to estimate the melting point. Molecular dynamics simulations are used to calculate the pair distribution function, numbers of nearest neighbors and the translational order parameter, analyzing the weakening of the fcc-symmetry due to emerging premelting effects. A range of dynamic properties, such as mean-squared displacement, non-Gaussian parameter, Debye-Waller factor and the vibrational density of states, is considered for the analysis of the solid state. All of these parameters clearly show that bulk mobility is activated at about $2 / 3$ of the melting temperature, known as Tammann temperature. This indicates that vibrational motion of atoms is not maintained exclusively in the entire stable solid state and that collective atomic motion constitutes a precursor of the melting process.
\end{abstract}

Keywords: Lennard Jones fcc-solid, solid/fluid transition, melting precursors, Z method, pair distribution function, numbers of nearest neighbors, translational order parameter, mean-squared displacement, non-Gaussian parameter, Debye-Waller factor, vibrational density of states, Tammann temperature. 


\section{Introduction}

The melting process of crystals is a long-standing, highly debated issue of condensed matter physics. Its microscopic mechanism is not yet completely understood and a satisfactory theory is still missing. Normal melting is typically initiated at free surfaces or defects as a heterogeneous process occuring at the melting temperature $T_{\mathrm{m}}$. A thermodynamic criterion for determining $T_{\mathrm{m}}$ is the equality of the Gibbs energy of orthobaric solid and fluid phases. However, under ideal conditions, melting can progress homogeneously from the interior of a surface-free, perfect crystal. In such a situation, superheating of the crystal well above its equilibrium melting temperature is possible. Then, the phase transition to the fluid state starts from a metastable solid state at the limit of superheating (LS), characterized by the temperature $T_{\mathrm{LS}}$.

Several stability theories have been developed to interpret the melting mechanism. Lindemann [1] proposed a theory in which the vibration of atoms reaches a critical fraction of the nearest neighbor distance. Born [2] suggested that melting occurs when one of the shear moduli of the system vanishes. Jin et al. [3] were able to connect the ideas of Lindemann and Born by analyzing clusters of defective atoms, so-called Lindemann particles, which exhibit strong spatial correlations. Furthermore, Fecht and Johnson [4] proposed an entropy catastrophe theory that is analogous to Kauzmann's paradox [5] for the glass transition. Other models deal with dislocation lines composed of defective atoms [6-8] that are frequently defined as those which do not have 12 nearest neighbors in a face-centered cubic (fcc) lattice. In several recent studies [9-12] melting was discussed as a diffusion-mediated process, exhibiting string-like cooperative atomic motion, which is a form of dynamic heterogeneity.

Many of the above-mentioned studies on the solid-fluid (S/F) transition focus on the 
superheated regime because various properties exhibit a specific anomalous behavior as they approach the LS. However, a study of Köster et al. [13] showed that the onset of accelerated "excitation" of many thermodynamic properties starts well below the melting temperature in the stable solid region and that has frequently been suggested as an indicator for premelting. This issue is briefly reviewed in the supplementary material, taking the isochoric heat capacity $c_{V}$ as an example (cf. Figs. S1 and S2). The thermodynamics of the considered state region was entirely described in Ref. [13], thus, a complementary study on the structure and dynamics of the Lennard-Jones (LJ) fcc-solid is of interest and the aim of the present study. It focuses on aspects of diffusion-mediated models [9-12] with a special consideration of melting precursors, reflecting anomalous behavior of thermodynamic response functions.

The investigations are supplemented by estimations of the LS, melting point (MP) and freezing point (FP). The so-called $\mathrm{Z}$ method is applied for this purpose, which has recently become widespread in the literature [10,14-17]. Assessments of finite size effects are carried out over a large range of atom numbers, which has not yet been done for the $\mathrm{Z}$ method. In addition, the FP is determined with the Hansen-Verlet criterion.

The paper is organized as follows: First, the molecular simulation method for the analysis and its associated parameter settings is introduced. A discussion of the Z method estimating the LS and the MP follows. Next, structural changes of the fcc-solid as it approaches the S/F transition are examined. Subsequently, the dynamic behavior is elucidated. Conclusions sum up the findings. 


\section{Molecular simulation method}

The present study is limited to atoms interacting via the LJ potential commonly expressed as

$$
u_{\mathrm{LJ}}=4 \varepsilon\left[\left(\frac{\sigma}{r}\right)^{12}-\left(\frac{\sigma}{r}\right)^{6}\right]
$$

where $\sigma$ and $\varepsilon$ are size and energy parameters, while $r$ is the distance between two atoms. The usual conventions were used for the reduced density $\left(\rho^{*}=\rho \sigma^{3}\right)$, temperature $\left(T^{*}=\right.$ $\left.k_{\mathrm{B}} T / \varepsilon\right)$, energy $\left(u^{*}=u / \varepsilon\right)$, distance $\left(r^{*}=r / \sigma\right)$ and time $\left(t^{*}=t \sqrt{\varepsilon / m \sigma^{2}}\right)$, with Boltzmann's constant $k_{\mathrm{B}}$. The reduced form of the other discussed properties is: translational order parameter $\left(\tau^{*}=\tau / \sigma\right)$, mean-squared displacement $\left(\left\langle\Delta r^{* 2}(t)\right\rangle=\left\langle\Delta r^{2}(t)\right\rangle / \sigma^{2}\right)$, Debye-Waller factor $\left(\left\langle u^{* 2}\right\rangle=\left\langle u^{2}\right\rangle / \sigma^{2}\right)$, self-diffusion coefficient $\left(D^{*}=D \sqrt{m / \varepsilon \sigma^{2}}\right)$ and vibrational density of states $\left.\rho_{\mathrm{V}}^{*}=\rho_{\mathrm{V}} \sqrt{\varepsilon / m \sigma^{2}}\right)$. Pair distribution function $(g(r))$, nonGaussian parameter $\left(\alpha_{2}(t)\right)$ and normalized velocity autocorrelation function $(\psi(t))$ are dimensionless by definition. All quantities quoted in this work are given in terms of these reduced quantities so that the asterisk superscript will be omitted in the remainder.

Throughout the paper, molecular dynamics (MD) simulations were performed, integrating Newton's equations of motion with a fifth-order Gear predictor-corrector scheme by using the molecular simulation tool $m s 2$ [18-20]. The microcanonical (NVE) ensemble was employed for the $\mathrm{Z}$ method, whereas all other simulation data were sampled in the canonical (NVT) ensemble. Since long-range effects are important in solids, the atomic interactions were explicitly evaluated up to a large cutoff radius $r_{\mathrm{c}}=9.1$ in all NVT simulations. Beyond that distance, analytical long-range corrections were applied. To exclude vacancy effects, all simulations were initiated from a perfect fcc-crystal. Consequently, with a cubic simulation volume, the atom number must adhere to $N=4 i^{3}$, where $i$ is an integer. Simulations were performed along the isochore $\rho=1.8$ for temperatures ranging 
from $T=1$ to 26 . The isochore $\rho=1.8$ was chosen in order to cover a large region with anomalous behavior (cf. Fig. S2 in the supplementary material).

For each state point, the system was sufficiently equilibrated and then sampled during the production period. As a result of the finite size investigations in section 3 , an atom number $N=10976$ was used for most simulations. The specific simulation parameters (such as the cutoff radius $r_{\mathrm{c}}$, time step $\Delta t$ or simulation length) were adapted to the requirements of the function to be determined and are compiled in Table S1 of the supplemental material.

\section{$3 \quad \mathrm{Z}$ method and finite size effects}

Methods for determining the S/F phase transition can roughly be classified in two groups. The first group comprises phenomenological procedures based on atomistic simulations revealing a specific behavior of a first-order phase transition. This includes, e.g., the evaluation of an equation of state (EOS) that is employed to identify discontinuous jumps or hysteresis loops as a hallmark of phase transition. Other approaches test the phase equilibrium conditions with direct simulations of an inhomogeneous system containing the two coexisting phases separated by an interface. In the second group, methods are applied that explicitly evaluate the Gibbs energy of the system, which allows for a clear localization of coexistence between the two phases.

In the present study, a phenomenological method was applied which determines the $\mathrm{S} / \mathrm{F}$ transition starting from the solid state. The so-called $\mathrm{Z}$ method allows for an estimation of the LS and the MP. In order to reach the LS, the system has to evolve on its own without external interference into the dynamics of the melting process as, e.g., by constraining the temperature. Therefore, MD simulations in the NVE ensemble have to be applied $[10,14]$. The idea of the $\mathrm{Z}$ method is to equilibrate the system to some 
temperature at a given total energy. In a certain total energy range, the crystal resides in a metastable solid state. That range is delimited by $u_{\mathrm{LS}}$, where the crystal spontaneously melts at $T_{\mathrm{LS}}$. A total energy slightly above $u_{\mathrm{LS}}$ leads to a temperature drop to the melting temperature $T_{\mathrm{m}}$ because the kinetic energy has to supply the internal energy of fusion. At this point, the total energies of the solid at $T_{\mathrm{LS}}$ and the fluid at $T_{\mathrm{m}}$ are assumed to be equal if the volume/density was conserved $[10,14]$

$$
u^{\text {solid }}\left(\rho, T_{\mathrm{LS}}\right)=u^{\text {fluid }}\left(\rho, T_{\mathrm{m}}\right) .
$$

Fig. 1 depicts time dependence of total energy $u$ and temperature $T$ at four state points, calculated with an atom number $N=10976$. These state points are marked in the $(u, T)$ representation of Fig. 2 by red arrows 1 to 4 . Within the scope of usual simulation accuracies, the total energy remains constant over the entire simulation period (note the very magnified scale of $u$ ). State point 1 starts with $u=84.00025$ and resides in a metastable solid state with constant temperature. State point 2 with $u=87.00025$ is just below $u_{\mathrm{LS}}$, being still in the metastable solid. At $u=u_{\mathrm{LS}}=87.25025$ (state point 3) the temperature lingers for a certain time at the LS, then drops to the melting temperature $T_{\mathrm{m}}$ and then remains constant. At state point 4 with a total energy of $u=89.00025>u_{\mathrm{LS}}$, the temperature drops almost immediately towards a fluid state temperature.

Collecting $(u, T)$ data obtained from a series of NVE simulations results in a Z shaped curve, where the maximum temperature corresponds to $T_{\mathrm{LS}}$ and that at the local minimum to $T_{\mathrm{m}}$. Since studies on finite size effects related to the $\mathrm{Z}$ method are rare in the literature, attention is given to this issue. For this purpose, simulations were performed for atom numbers $N=256,500,1372,4000,10976,32000$ and 108000 , all initially arranged as a perfect fcc-crystal. 


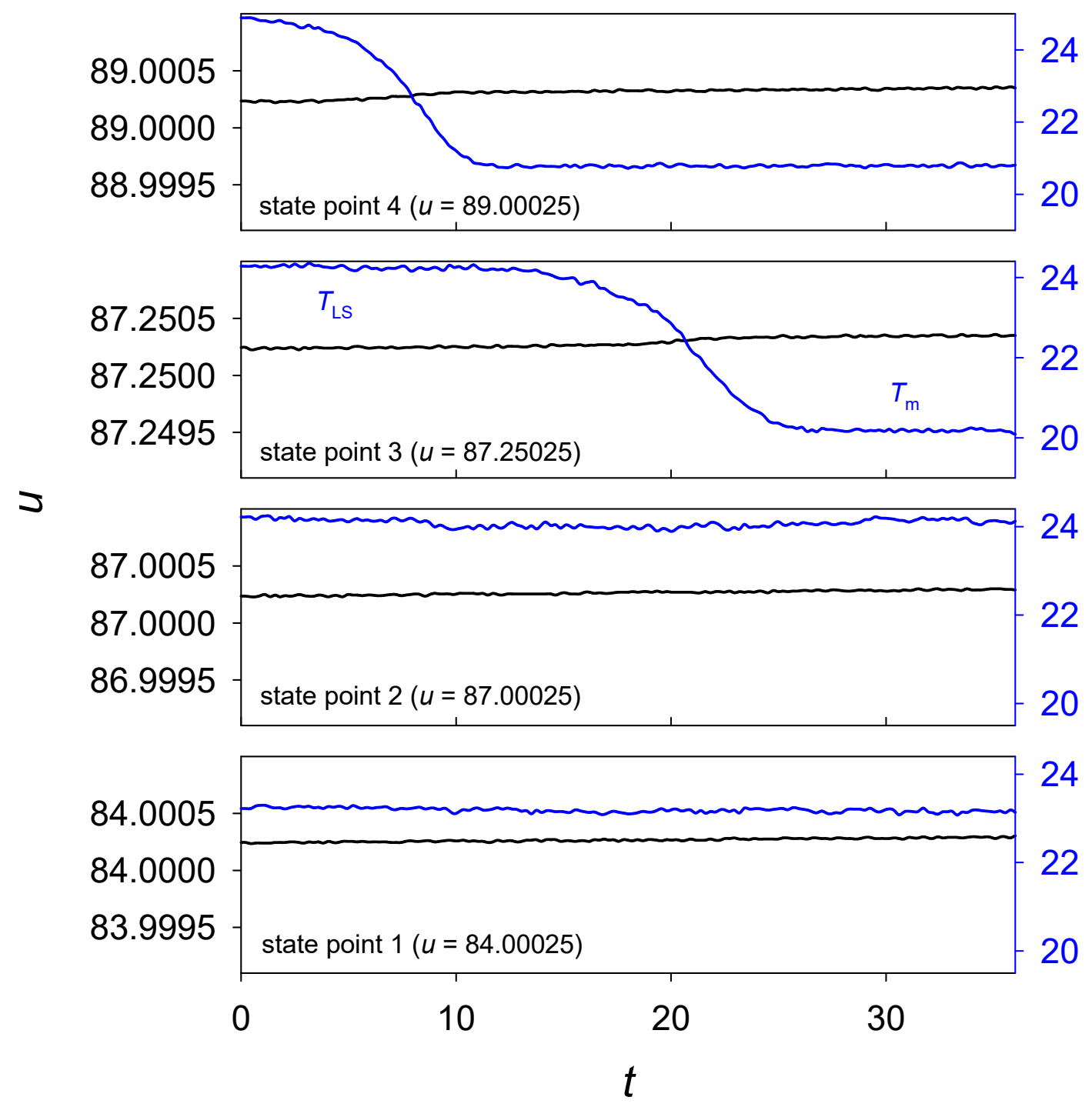

Figure 1: Instantaneous total energy $u$ (black) and temperature $T$ (blue) as a function of time $t$ obtained from NVE simulations with an atom number $N=10976$. The left vertical axis shows the scale for $u$ and the right vertical axis shows the scale for $T$. Four different phase points are shown, 1 and 2 belong to the metastable solid state, 3 is at the LS and 4 is in the fluid state. 


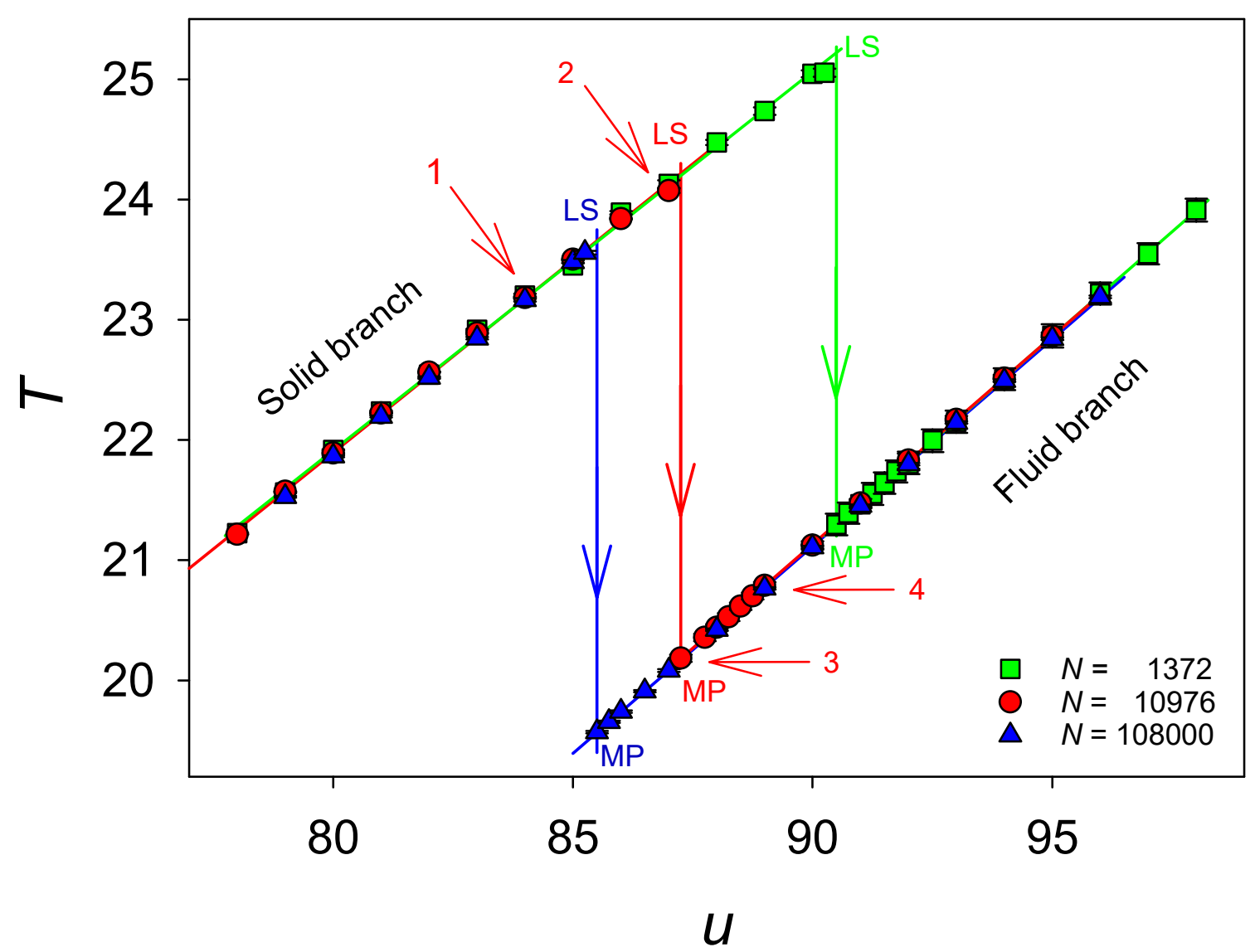

Figure 2: Temperature $T$ as a function of total energy $u$ obtained from NVE simulations with the $\mathrm{Z}$ method. Results for different atom numbers $N=1372,10976$ and 108000 along the isochore of $\rho=1.8$ are shown. The upper curves belong to the solid branch, the lower curves to the fluid branch. Red arrows 1 to 4 mark the four state points discussed in Fig. 1. Vertical lines indicate the temperature drop from the LS to the MP according to Eq. (2). 


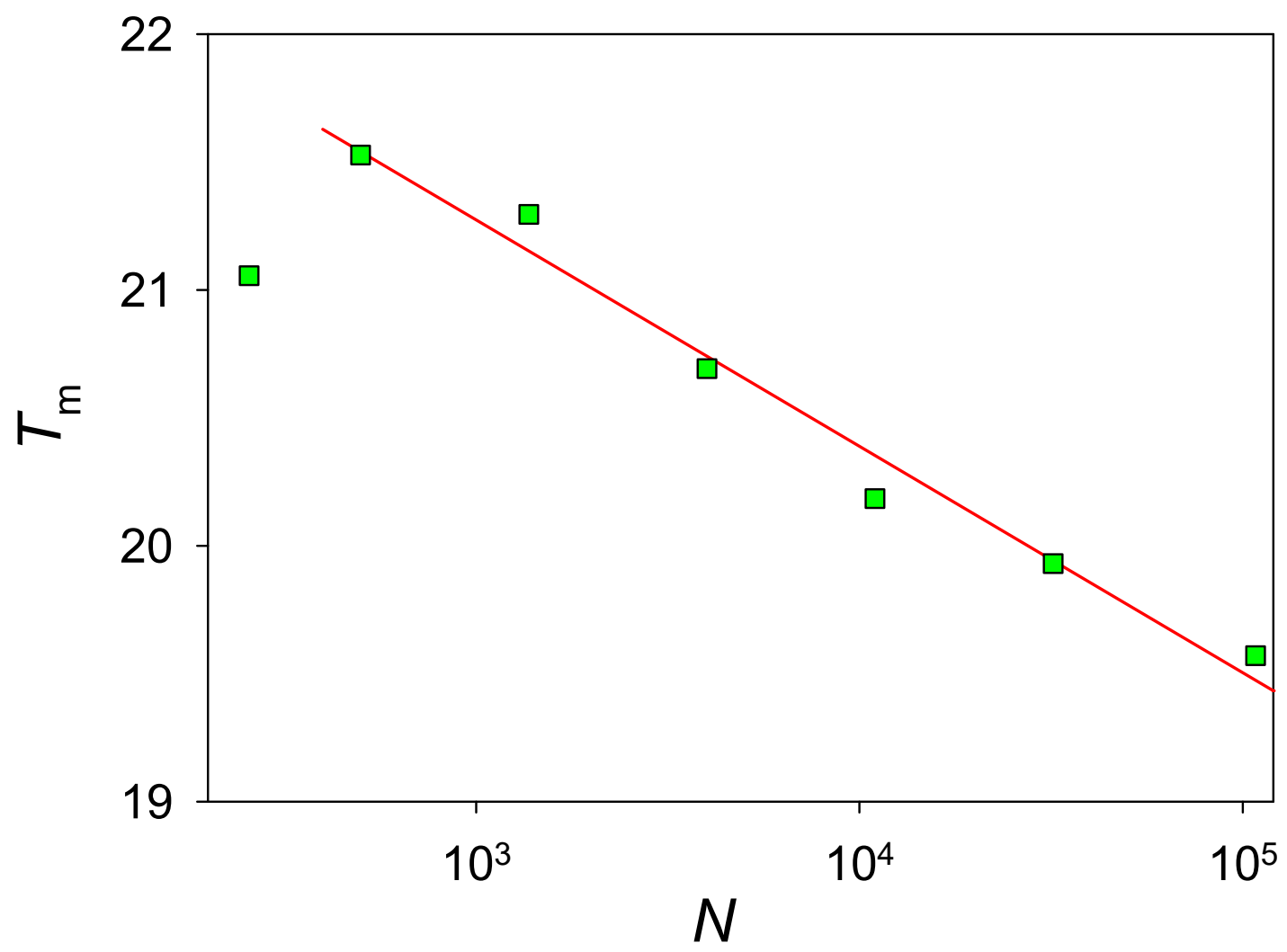

Figure 3: Melting temperature $T_{\mathrm{m}}$ as a function of system size $N$ along the isochore $\rho=1.8$. $T_{\mathrm{m}}$ was obtained from the $\mathrm{Z}$ method according to Fig. 2 and Fig. $\mathrm{S} 3$ in the supplemental material. The red line is a power-law fit for system sizes between $N=500$ and 108000 . 
For clarity, Fig. 2 shows typical results for $N=1372,10976$ and 108000 , other atom numbers are discussed in the supplemental material (Fig. S3). Eq. (2) is satisfied along the vertical lines in Fig. 2. Interestingly, the MP and LS show a rather strong dependence on the atom number, which becomes even clearer when the melting temperature $T_{\mathrm{m}}$ is shown as a function of $N$, cf. Fig. 3. For $N \geq 500$, the melting temperature decreases with rising atom number $N$, roughly following a power law. Obviously, an approach to an asymptotic limit of the melting temperature cannot be achieved for atom numbers analyzed in this study. This corresponds to the behavior of the melting temperature of silicon examined in a study by Nieves and Sinno [21] for a large range of particle numbers between $N=2744$ and 238328. Nieves and Sinno concluded that finite size effects intrinsically extend to rather large system sizes because critical defect clusters are not well sampled unless the system size is very large. Therefore, additional superheating must be provided for smaller system sizes in order to initiate crystal melting. Results that support these findings have been published by Bai and $\mathrm{Li}$ [22], who examined the critical liquid nucleus radius of a superheated LJ crystal. Finite size investigations for $N=32000,108000$ and 256000 showed a strong size dependence. Aspects like these have not yet been properly investigated for the $\mathrm{Z}$ method.

In another study, Abramo et al. [23] analyzed the system size dependence of the S/F phase transition for a LJ system between $N=108$ and 4000 , using a phenomenological method comparable to that employed in Ref. [13]. The authors concluded that with small atom numbers, specifically $N=108$, the best agreement with respect to the location of the freezing line and melting line can be found. However, this statement cannot be confirmed on the basis of the present results obtained with the $\mathrm{Z}$ method.

Although finite size effects are important for the determination of the S/F transition, their quantitative influence is rather small for the thermodynamic properties when $N \geq$ 
1372. This can be recognized, for example, from the almost congruent temperature curves $T=T(u)$ in Fig. 2 and Fig. S1 in the supplemental material as well as from other properties [13]. This is an important result since the present study focuses on melting precursors, appearing well below the MP. The LS serves as an upper bound of the metastable solid state and reaching it truly is not of central importance here. However, the atomistic ensemble should be sufficiently large, at least larger than typical structural inhomogeneities arising when the MP is approached. Therefore, in the remainder of the present study, $N=10976$ was chosen.

The present estimation of the MP and LS is supplemented by the determination of the FP at $\rho=1.8$. The Hansen-Verlet criterion [24] was applied, where freezing is indicated if the magnitude of the first peak of the fluid structure factor reaches a value of 2.85. The criterion is fulfilled for $T_{\mathrm{f}}=24.775$, details are discussed in the supplemental material.

In summary, the results of the present study for the temperatures determining the $\mathrm{S} / \mathrm{F}$ transition at $\rho=1.8$ are $T_{\mathrm{m}} \approx 20.18, T_{\mathrm{LS}} \approx 24.2$ and $T_{\mathrm{f}} \approx 24.775$.

\section{Structure}

The melting process of a crystal can be thought of as an order-disorder transition in which the crystal loses its long-range translational order. In this context, the number of first nearest neighbors $(1 \mathrm{NN})$ is a basic order parameter. Since for a perfect fcc-crystal $1 \mathrm{NN}=$ 12 , any deviation from this number indicates structural changes in the crystal symmetry. For example, a missing neighboring atom leaves free volume in the lattice structure with important consequences for the melting behavior. Other defects arise when atoms are located on interstitial sites, pushing the surrounding atoms out of their equilibrium lattice positions. The key function for examining $1 \mathrm{NN}$, or any other number of neighbors from which defective behavior may be observed, is the pair distribution function (PDF). 


\subsection{Pair distribution function}

The PDF $g(r)$ was sampled along the isochore $\rho=1.8$ in a temperature range from $T=1$ to 26 . Fig. 4 depicts a 3D representation of the PDF up to an atom-atom distance of $r \leq 1.95$. The solid phase is characterized by sharp peaks around the lattice sites, which are increasingly smeared out as the temperature rises. The magnitude of the peaks decreases drastically with raising temperature. Moreover, the second and fourth peak almost disappear within the stable solid phase, weakening the fcc geometry. In a relatively narrow temperature range from $T=22$ to 23 , the PDF rapidly smoothens, indicating the transition from the crystal to the fluid state. Within the fluid state, the second and fourth peaks of the solid phase PDF disappear completely .

In order to localize the transition temperature from the solid to the fluid phase more precisely, PDF are shown in Fig. 5 ranging from $T=22$ to 22.6 . The PDF at $T=22$ and 22.2 as well as at $T=22.4$ and 22.6 are almost congruent in Fig. 5 so that the S/F transition was identified to be between $T=22.2$ and 22.4 .

\subsection{Running coordination number and nearest neighbors}

The running coordination number

$$
n(r)=4 \pi \rho \int_{0}^{r} r^{\prime 2} g\left(r^{\prime}\right) d r^{\prime}
$$

allows for the determination of the number of neighbors in spherical shells around a reference atom. For the considered fcc-solid, $n(r)$ is depicted in Fig. S7 in the supplemental material. Defining the $j$ th spherical shell around the $j$ th peak between the $(j-1)$-th and $j$ th minima $\left(r_{j-1, \min }, r_{j, \text { min }}\right)$ of the PDF, the number of next neighbors in this shell is given by $j \mathrm{NN}=n\left(r_{j, \min }\right)-n\left(r_{j-1, \min }\right)$, with minimum positions $r_{j, \min }$ compiled in Table 1 and visualized in Fig. S8 in the supplemental material. Relying on this definition, the number of atoms in each shell may change with temperature and density, which allows 


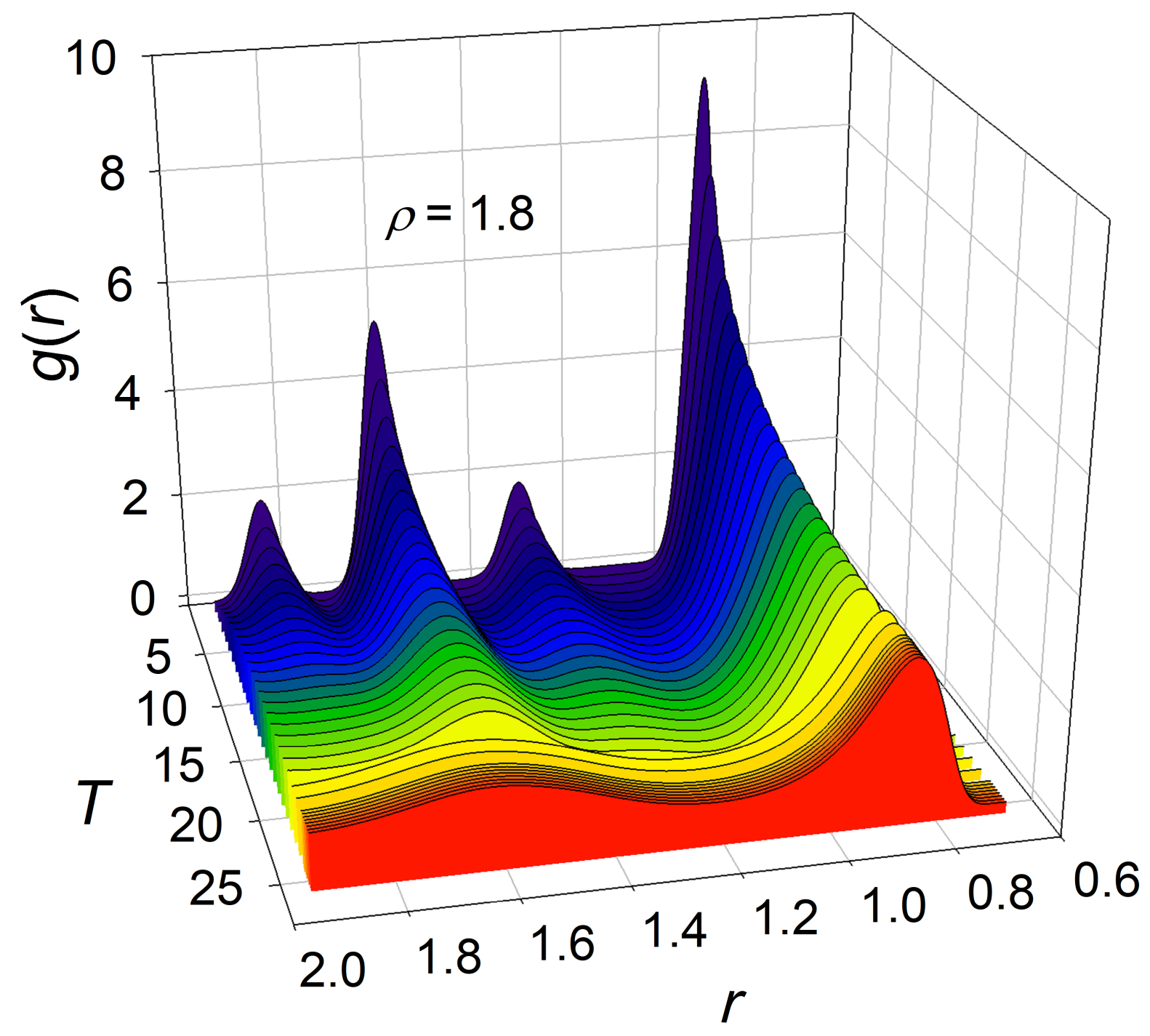

Figure 4: 3D representation of the of the first four PDF peaks along the isochore $\rho=1.8$ for $T=1$ to 26 . Upon the approach of the MP, the second and fourth peaks disappear. The $\mathrm{S} / \mathrm{F}$ transition region is yellow, the fluid phase is red. 


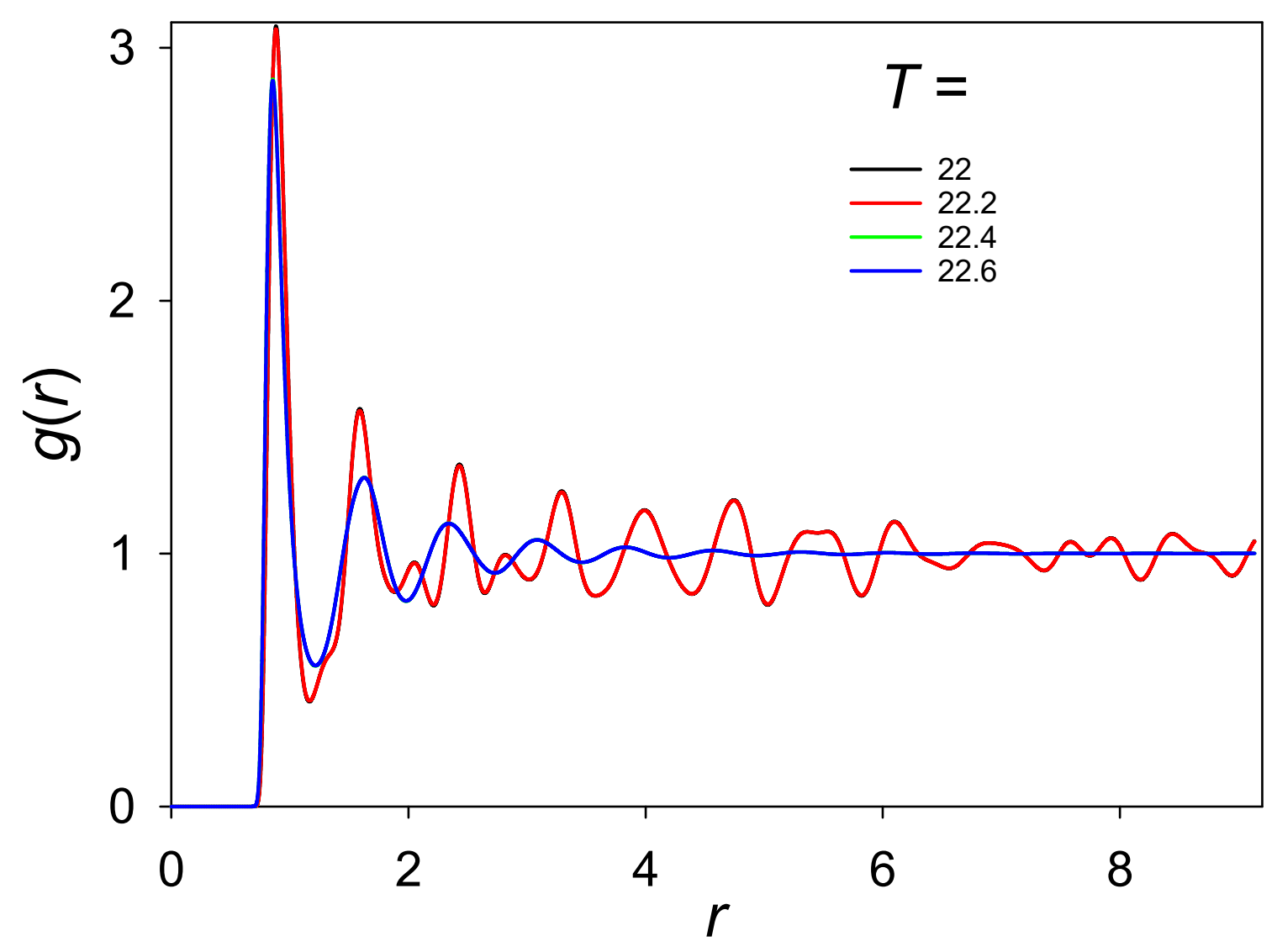

Figure 5: Pair distribution function $g(r)$ along the isochore $\rho=1.8$ for $T=22$ to 22.6. Due to the very small numerical differences between the data for $T=22$ and 22.2 as well as for $T=22.4$ and 22.6, the black line is behind the red line and the green line behind the blue line. 
Table 1: Minima separating the peaks of the PDF at $T=1$ and $\rho=1.8$.

\begin{tabular}{c|c}
\hline$j$ & $r_{j, \min }$ \\
\hline 0 & 0 \\
1 & 1.1108 \\
2 & 1.4524 \\
3 & 1.7283 \\
4 & 1.9512 \\
\hline
\end{tabular}

for statements about the lattice order. Next neighbors $1 \mathrm{NN}$ and $2 \mathrm{NN}$ are shown in Fig. 6, next neighbors 3NN and 4NN are shown in Fig. S9 in the supplemental material.

Studying the microscopic mechanism of LJ fcc-crystal melting, Gómez et al. [7] investigated dislocation lines, suggesting that the appearance of these lines constitutes a precursor of the melting process. At temperatures above $\sim 0.8 T_{\mathrm{m}}$, thermal fluctuations and distortions should create defective atoms, clustering into dislocation loops when these atoms become neighbors of each other. Knowing the individual coordination numbers, it is possible to identify under-coordinated and over-coordinated atoms as point defects. Gómez et al. defined a defective atom when its number of first nearest neighbors 1NN is not 12 .

The course of $1 \mathrm{NN}$ in Fig. 6 evidences a systematic and gradual reduction of the number of first nearest neighbors with increasing temperature, dropping from 11.7 to 11.2 at the $\mathrm{S} / \mathrm{F}$ transition. Therefore, a large number of atoms must be individually under-coordinated, which confirms the findings of Gómez et al. [7]. Furthermore, 2NN increases from 6 to 7.4 near the $\mathrm{S} / \mathrm{F}$ transition and jumps to 8.9 in the fluid phase. Thus, in the solid phase, on average more than one atom migrates into the $2 \mathrm{NN}$ shell due to isochoric heating, while its peak structure is lost. Fig. 6 shows the present data for MP, LS and FP in comparison to MP/FP coordinates from the literature. As a general trend, the FP agrees well for a number of studies. The FP coordinates of Köster 


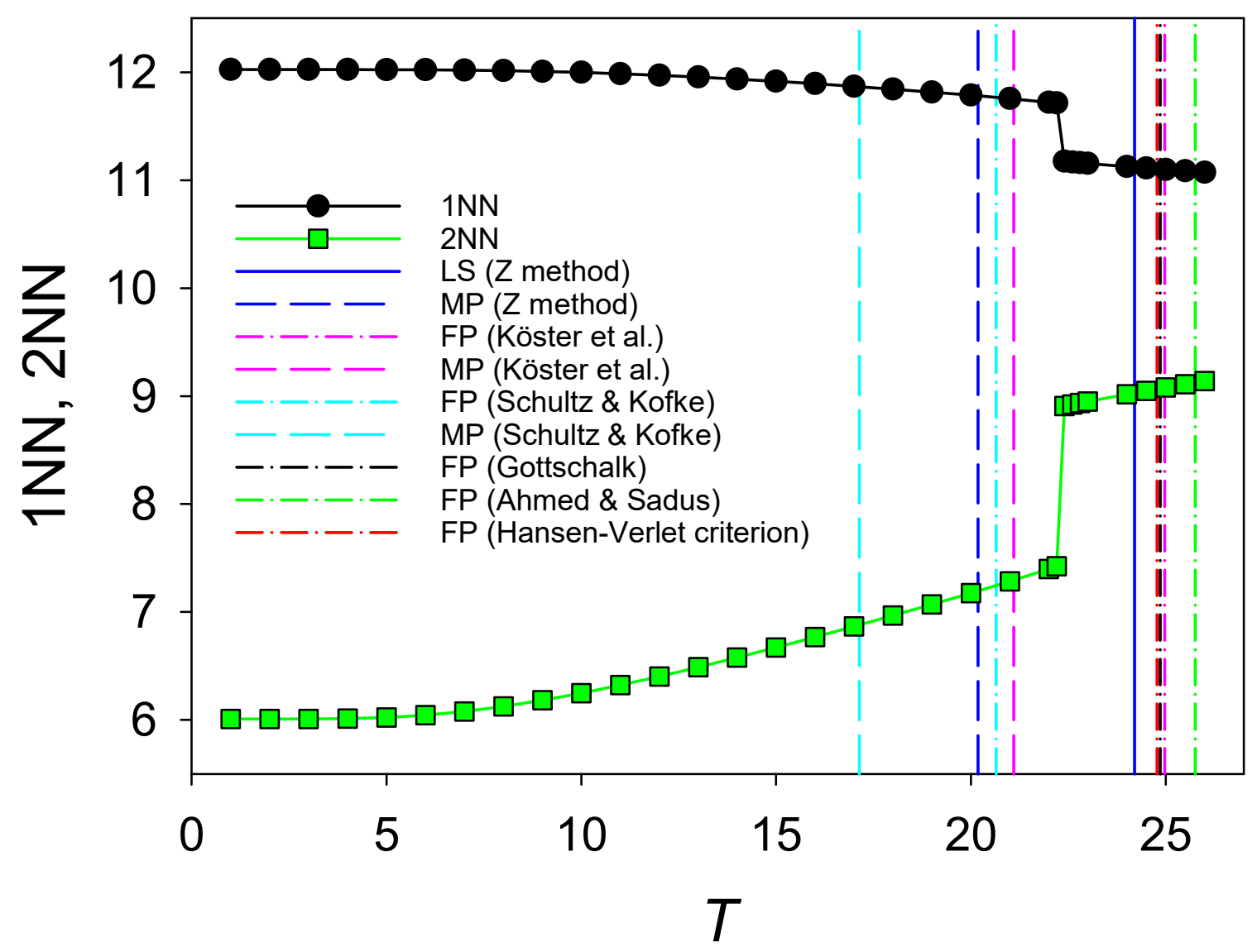

Figure 6: Temperature dependence of next nearest neighbors $1 \mathrm{NN}$ and $2 \mathrm{NN}$ along the isochore $\rho=1.8$ for $T=1$ to 26 . The vertical blue dashed line marks the MP, the blue solid line the LS calculated with the $\mathrm{Z}$ method and the red vertical dashed dotted line marks the FP according to the Hansen-Verlet criterion (cf. supplemental material). All data were generated with $N=10976$. Additionally shown is the FP from Gottschalk [25] and Ahmed and Sadus [26] as well as the FP/MP from Köster et al. [13] and Schultz and Kofke [27]. 
et al. [13], Gottschalk [25] and the results according to the Hansen-Verlet criterion are almost identical at $T \approx 25$. The FP of Ahmed and Sadus [26] is also close to this value. The situation is different for the MP, where the associated values vary over a larger temperature range. Note that the $\mathrm{S} / \mathrm{F}$ transition drop of next nearest neighbors $1 \mathrm{NN}$ and $2 \mathrm{NN}$ occurs before the LS (blue vertical solid line in Fig. 6 with $T_{\mathrm{LS}} \approx 24.2$ for $N=10976)$. This indicates that the LS can not be reached with the NVT ensemble and confirms that the NVE ensemble needs to be applied to estimate the LS [10]. For clarity, only MP, LS and FP data obtained in this study are shown in the following figures.

Another interesting aspect can be seen in Fig. 7 that depicts the temperature dependence of $g(r)$ for the first four peaks. The arrow at the top left marks the S/F transition temperature. The rapid disappearance of the second and fourth peaks can clearly be seen. More interesting is the fact that the $1 \mathrm{NN}$ shell is compressed during the isochoric temperature rise (the first peak moves to smaller interatomic distances), while the position of the other three peaks is fairly independent on temperature. Considering the interstitial, atom-free interval $1.034<r<1.189$ between the first and second peak at $T=1$, it was observed that this shell is filled up with atoms when the temperature rises. Fig. 8 shows the temperature dependence for $n_{\text {int }}(1 p, 2 p)=n(1.189)-n(1.034)$. Near the MP, on average about two atoms migrate into this interstitial shell, compressing the $1 \mathrm{NN}$ shell.

\subsection{Translational order parameter}

The strong increase of $2 \mathrm{NN}$ with a simultaneous loss of the peak structure shows that the number of nearest neighbors $j \mathrm{NN}$ is not a good quantity to assess the translational order of a system. Errington et al. [28] introduced a metric that quantifies the deviation of an actual structure from a reference arrangement by 


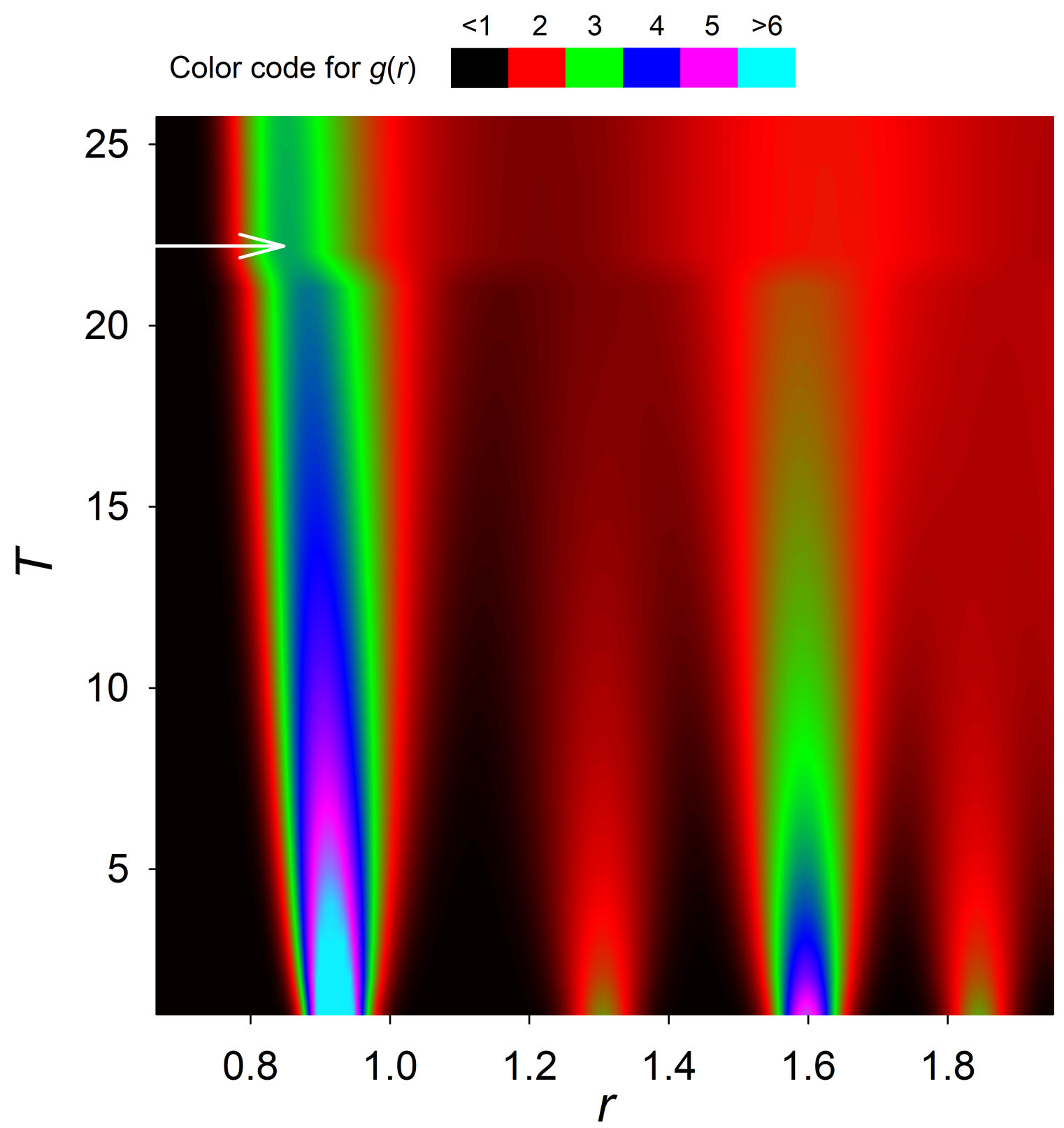

Figure 7: Contours of the temperature dependence of $g(r)$ along the isochore $\rho=1.8$ for $T=1$ to 26 . The arrow marks the $\mathrm{S} / \mathrm{F}$ transition. 


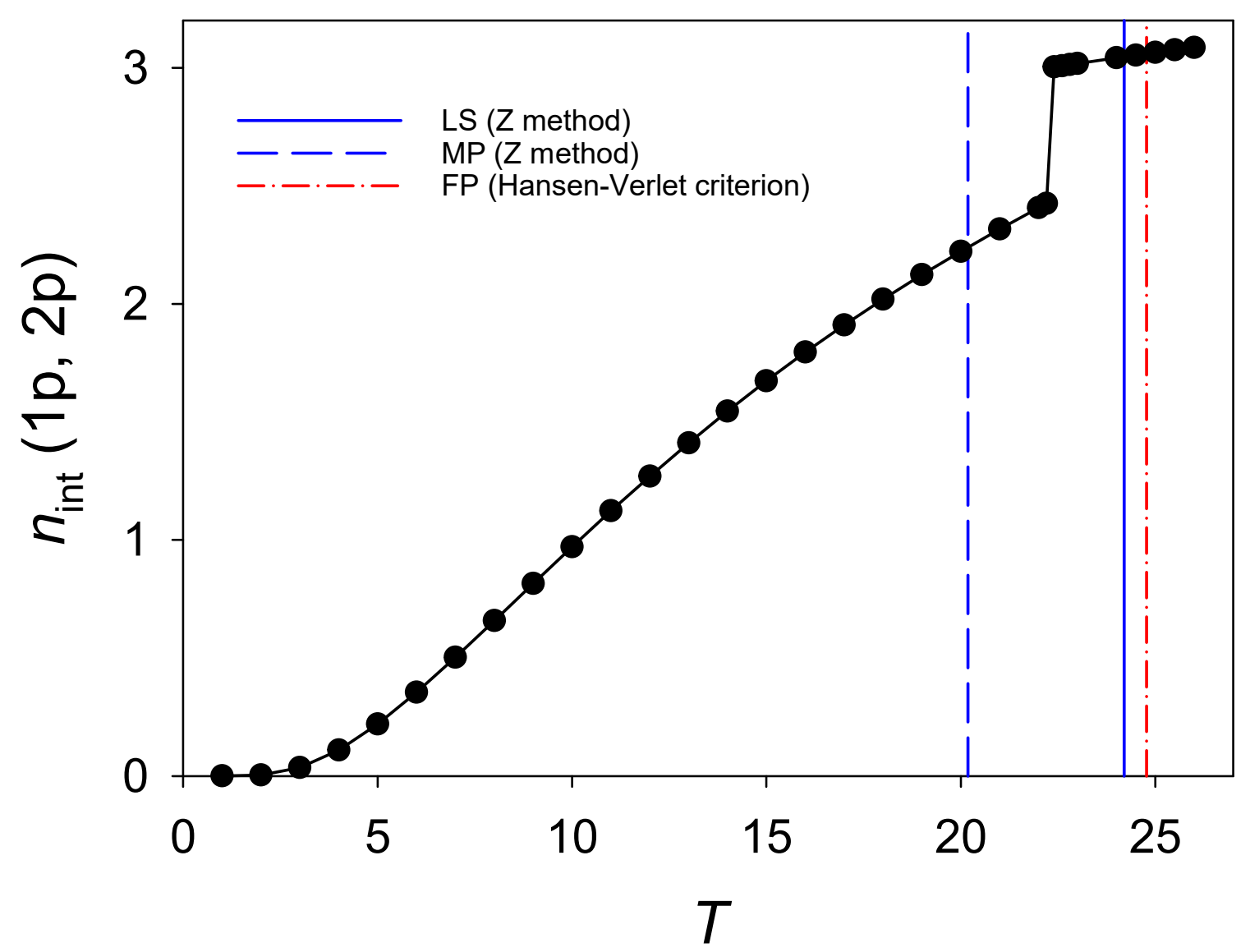

Figure 8: Temperature dependence of the atom number $n_{\text {int }}$ within the interstitial spherical shell $1.034<r<1.189$ between the first and second peak of the PDF. Symbols and conditions are the same as in Fig. 6. 


$$
\tau=\int_{0}^{r_{c}}|g(r)-1| d r
$$

where $r_{c}$ corresponds to a large cutoff radius. A completely uncorrelated system $(g(r)=1)$ gives $\tau=0$, while a crystal with long-range order exhibits large $\tau$ values. The translational order parameter $\tau$ can be decomposed into $\tau_{1}, \tau_{2}, \tau_{3}, \ldots$ by integrating $|g(r)-1|$ over the sections $0<r<r_{1, \min }, r_{1, \min }<r<r_{2, \min }, r_{2, \min }<r<r_{3, \min }, \ldots$ so that $\tau=\tau_{1}+\tau_{2}+$ $\tau_{3}+\ldots$. Fig. 9 shows the temperature dependence of $\tau_{1}, \ldots, \tau_{4}$ and $\tau=\tau_{1}+\tau_{2}+\tau_{3}+\tau_{4}$. As expected, all parameters decrease with raising temperature, indicating a gradual loss of translational order. Similar to other properties, a strong drop can be observed at the transition to the fluid state. The order parameters of the second and fourth shell, $\tau_{2}$ and $\tau_{4}$, decrease rapidly even at relatively low temperatures so that they are only weakly dependent on temperature for $T>10$. In contrast, $\tau_{1}, \tau_{3}$ and $\tau$ decrease without this characteristic.

In summary, restructuring within various shells, starting in the stable solid region far away from the melting point, was observed. It is caused by activated diffusion as discussed in the following section.

\section{Dynamic properties}

Several recent studies examined correlated diffusion processes near the S/F transition, focusing on the region close to the LS. Delogu [9] showed that melting of a superheated LJ fcc-crystal originates from defectively coordinated atoms arranged in pseudolinear clusters. The superheated crystal is, in this picture, dynamically heterogeneous, whereby defective atoms have a greater mobility than normally (12-fold) coordinated atoms. Belonoshko et al. [10] also studied the occurrence of diffusion and defects in a fcc-solid, concluding that melting is likely to be associated with the formation of elongated struc- 


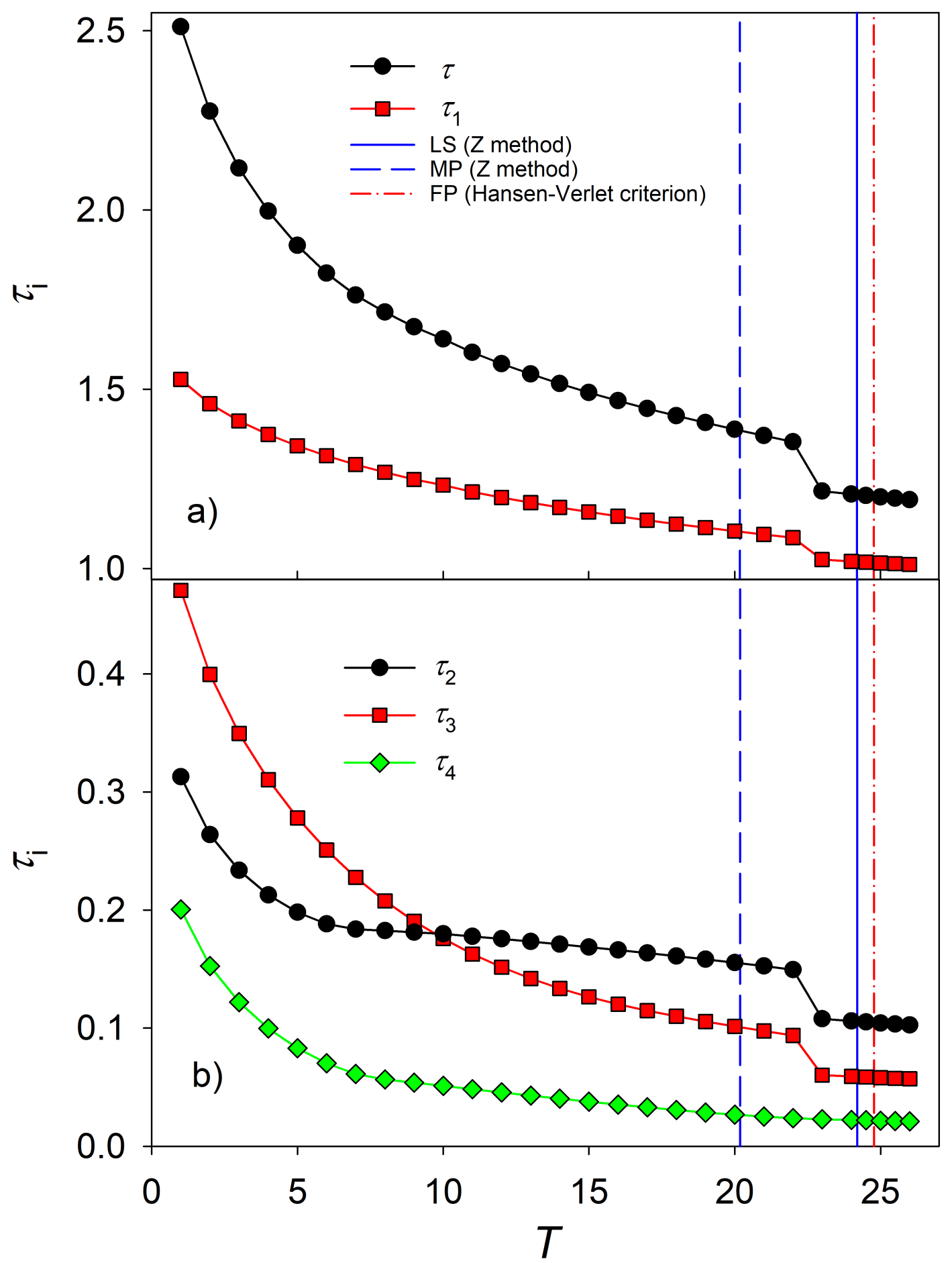

Figure 9: Temperature dependence of translational order parameters $\tau$ and $\left.\tau_{1}(\mathrm{a})\right)$ as well as $\tau_{2}, \tau_{3}$ and $\left.\tau_{4}(\mathrm{~b})\right)$. Symbols and conditions are the same as in Fig. 6. 
tures of dislocations. Common to these dynamical approaches is that string-like collective atomic motion occurs where groups of atoms follow each other along one-dimensional paths. Bai and Li [11] observed rapid self-diffusive motion as a precursor of melting when the system is kept at a constant temperature below the LS. They suggest that two types of string-like collective motion drive the homogeneous melting process, i.e. open and closed strings forming linear or ring (polymeric) structures. Qualitative evidence was provided that open diffusion loops, rather than closed ones, are related to the homogeneous nucleation of the fluid phase. In another study, Zhang et al. [12] examined similarities of highly correlated motion in a superheated Ni crystal to those found in glass-forming liquids. They identified a topological transition at higher temperatures when ring-like atomic exchanges "open" to form linear chains of atomic motions, similar to strings in glass-forming liquids. Ring-like diffusion loops rather preserve the crystalline structure because of their permutational character. However, the open strings result in a local symmetry breaking effect, triggering the crystal to melt.

Usually, atoms hardly diffuse in a crystal without vacancies. The main mechanism that drives diffusion in a perfect crystal is the occurrence of thermally activated jumps between short-time vacant equilibrium lattice sites [29] arising, e.g., when a neighboring atom occupies an interstitial site [10]. The behavior of $\Delta g(r, T)=g(r, T)-g(r, T=1)$ shows how these movements become possible, as discussed in Fig. S10 in the supplemental material.

Literature studies on diffusion mediated melting focused on regions close to the LS. Nevertheless, it is also of interest to investigate whether such effects are already initiated in the stable solid state, as it is the case with premelting effects in terms of structure and thermodynamic properties [13]. 


\subsection{Mean-squared displacement}

A common quantity describing the dynamics of a system is the single atom mean-squared displacement (MSD) $\left\langle\Delta r^{2}(t)\right\rangle$, where $\Delta r^{2}(t)$ is the squared distance propagated by a given atom at time $t$ and the angular brackets denote the ensemble average. Fig. 10.a shows typical MSD for the solid state. The well-known ballistic regime with $\left\langle\Delta r^{2}(t)\right\rangle \sim t^{2}$ can be recognized at very short times. A crossover from ballistic to vibrational motion follows, where the MSD exhibits a well-defined plateau, reflecting oscillations of atoms around their equilibrium lattice positions.

For higher temperatures, an additional regime appears at longer times, where atoms escape from their equilibrium lattice sites due to diffusion, cf. Fig. 10.b. By analyzing distributions of the atom displacements, Bai et al. [11] and Zhang et al. [12] showed that atoms migrate over a distance corresponding to their first neighboring lattice sites. At longer times, the MSD approaches the Einstein relation of $\left\langle\Delta r^{2}(t)\right\rangle=6 D t$, where $D$ is the self-diffusion coefficient.

It was frequently observed that many crystals acquire sufficient energy for their bulk mobility above an onset temperature $T_{\mathrm{T}} \approx 2 T_{\mathrm{m}} / 3$, known as Tammann temperature [30,31]. For example, Zhang et al. [32] consider $T_{\mathrm{T}}$ as a premelting onset temperature at which accelerated dynamics can be observed. On the time scale shown in Fig. 10.b, diffusive motion is initiated at approximately $T \approx 17 \approx 0.75 T_{\mathrm{m}}$, indicating that pure vibrational motion is not maintained in entire stable solid state, as discussed in the more recent literature.

The intermediate vibrational regime disappears completely at a temperature of $T=$ 22.4 and the MSD exhibits fluid behavior for all temperatures above. Note that this is exactly the temperature where $\mathrm{S} / \mathrm{F}$ phase transition was observed in the PDF, cf. Fig. 5 . 


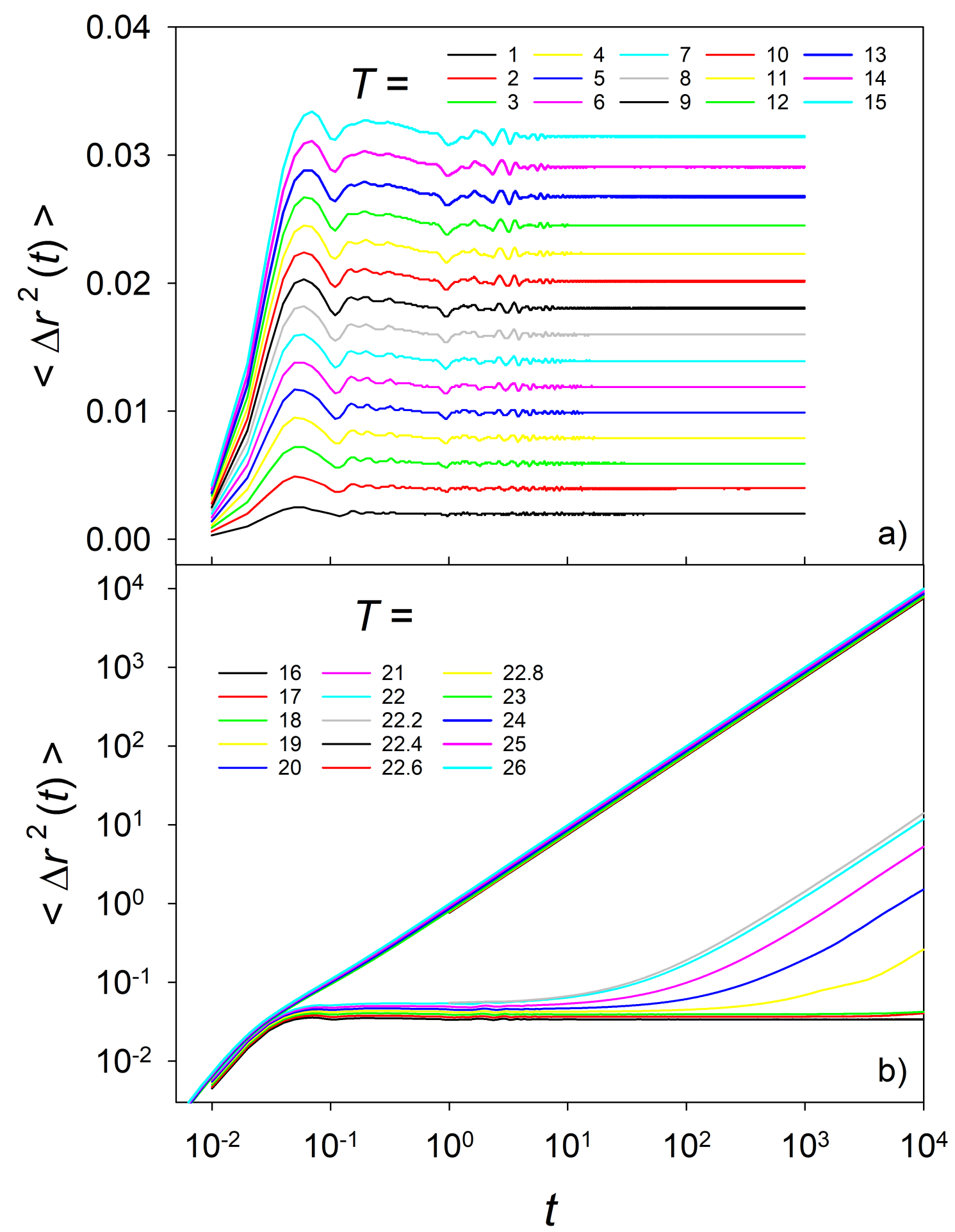

Figure 10: Mean-squared displacement $\left\langle\Delta r^{2}(t)\right\rangle$ as a function of time $t$ along the isochore $\rho=1.8$ for $T=1$ to 26 . Typical solid behavior can be observed up to a temperature of $T \approx 16$, consisting of a ballistic regime at very short times and a vibrational regime with a well-defined plateau for longer times (a)). An additional diffusive regime appears for $T \geq 17$ at longer times (b)). 


\subsection{Non-Gaussian parameter and Debye-Waller factor}

Dynamic heterogeneity caused by mobile and immobile atoms is commonly quantified by the non-Gaussian parameter (NGP)

$$
\alpha_{2}(t)=\frac{3\left\langle\Delta r^{4}(t)\right\rangle}{5\left\langle\Delta r^{2}(t)\right\rangle^{2}}-1
$$

It is known that the NGP mainly receives contributions from those atoms that move further than the Gaussian distribution of particle displacements. Consequently, $\alpha_{2}(t)$ has been suggested as an indicator for dynamical heterogeneity. Investigations based on the NGP should therefore allow for conclusions about mobile atoms, diffusing through a crystal consisting of immobile atoms. In case of ballistic and diffusive motion, displacements are known to follow a Gaussian distribution and consequently the NGP is zero in these regimes. This behavior can be recognized in Fig. 11.a for the fluid phase in the temperature range from $T=23$ to 26 . At the crossover between the two regimes, $\alpha_{2}$ develops a pronounced maximum. For the stable solid phase in a temperature range from $T=1$ to 16 , the behavior is different. The NGP starts at zero in the ballistic regime and then peaks at a time $t=0.1$, corresponding to the appearance of vibrational motion, followed by an approach to an almost constant plateau with a relatively small magnitude. At a temperature of $T=17$, the NGP rises again at longer times, comparable to the behavior of the MSD at this temperature.

Of greater interest is the behavior of $\alpha_{2}$ in the vicinity of the $\mathrm{S} / \mathrm{F}$ phase transition, cf. Fig. 11.b. The NGP is depicted there for temperatures from $T=17$ to 26 . A dramatic increase of $\alpha_{2}$ can be observed when the system is heated from $T=17$ to 19 , which signals the appearance of strongly heterogeneous dynamics. The maximum of $\alpha_{2}$ at $T=19$ corresponds to a characteristic time $t_{\max }$ when displacements are mostly non- 


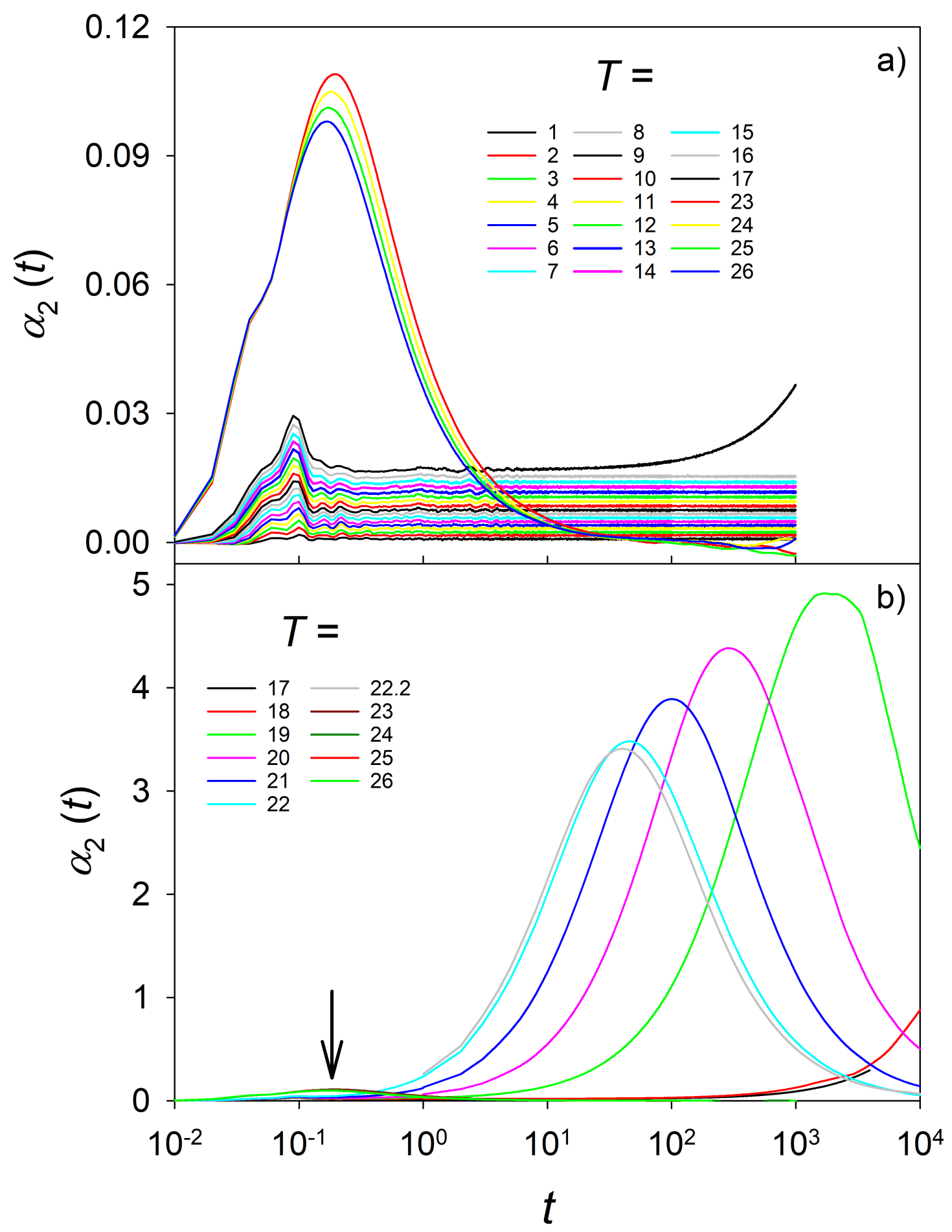

Figure 11: Non-Gaussian parameter $\alpha_{2}(t)$ as a function of time $t$ along the isochore $\rho=1.8$ for $T=1$ to $26(\mathrm{a}))$. In $\mathrm{b})$, the occurrence of the diffusive regime is characterized by pronounced peaks of $\alpha_{2}(t)$ between $T=19$ and 22.2 before the $\alpha_{2}(t)$ peak suddenly recedes at $T=23$, indicating the $\mathrm{S} / \mathrm{F}$ transition marked by the arrow. 


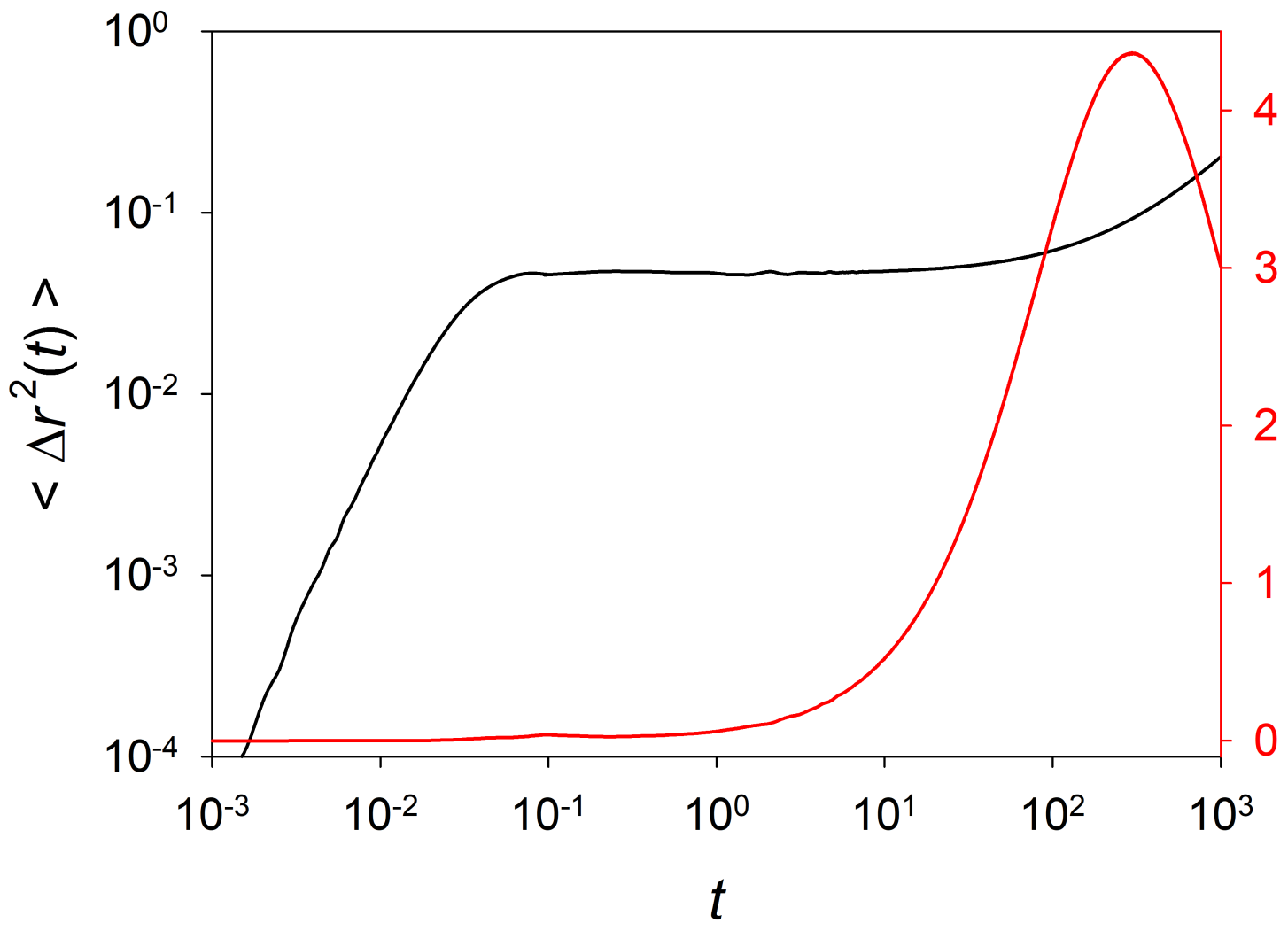

Figure 12: Mean-squared displacement $\left\langle\Delta r^{2}(t)\right\rangle$ (black) and non-Gaussian parameter $\alpha_{2}(t)$ (red) at $T=18.6$ and $\rho=1.768$. The scale for $\left\langle\Delta r^{2}(t)\right\rangle$ is given on the left axis, the scale for $\alpha_{2}(t)$ on the right axis.

Gaussian. Furthermore, the magnitude of $\alpha_{2}\left(t_{\max }\right)$ and $t_{\max }$ decreases from $T=19$ to 22.2. This is an indication that the transient vibrational regime becomes less pronounced as the atoms need less and less time to enter the diffusive regime.

The interplay between the MSD and $\alpha_{2}(t)$ is compared in Fig. 12 for the state point $T=18.6$ and $\rho=1.768$, which is close to the MP. The NGP peaks strongly at the transition from the vibrational to the diffusive regime, appearing roughly at $t_{\max }$ in the MSD. Consequently, the position of the peak $t_{\max }$ can be considered as an activation time of diffusion, where a single atom escapes from its equilibrium lattice position. Zhang et al. [12] argued that $t_{\max }$ has the significance of a characteristic diffusive relaxation time. From the behavior of $\left\langle\Delta r^{2}(t)\right\rangle=6 D t$ for long times, the self-diffusion coefficient $D$ 


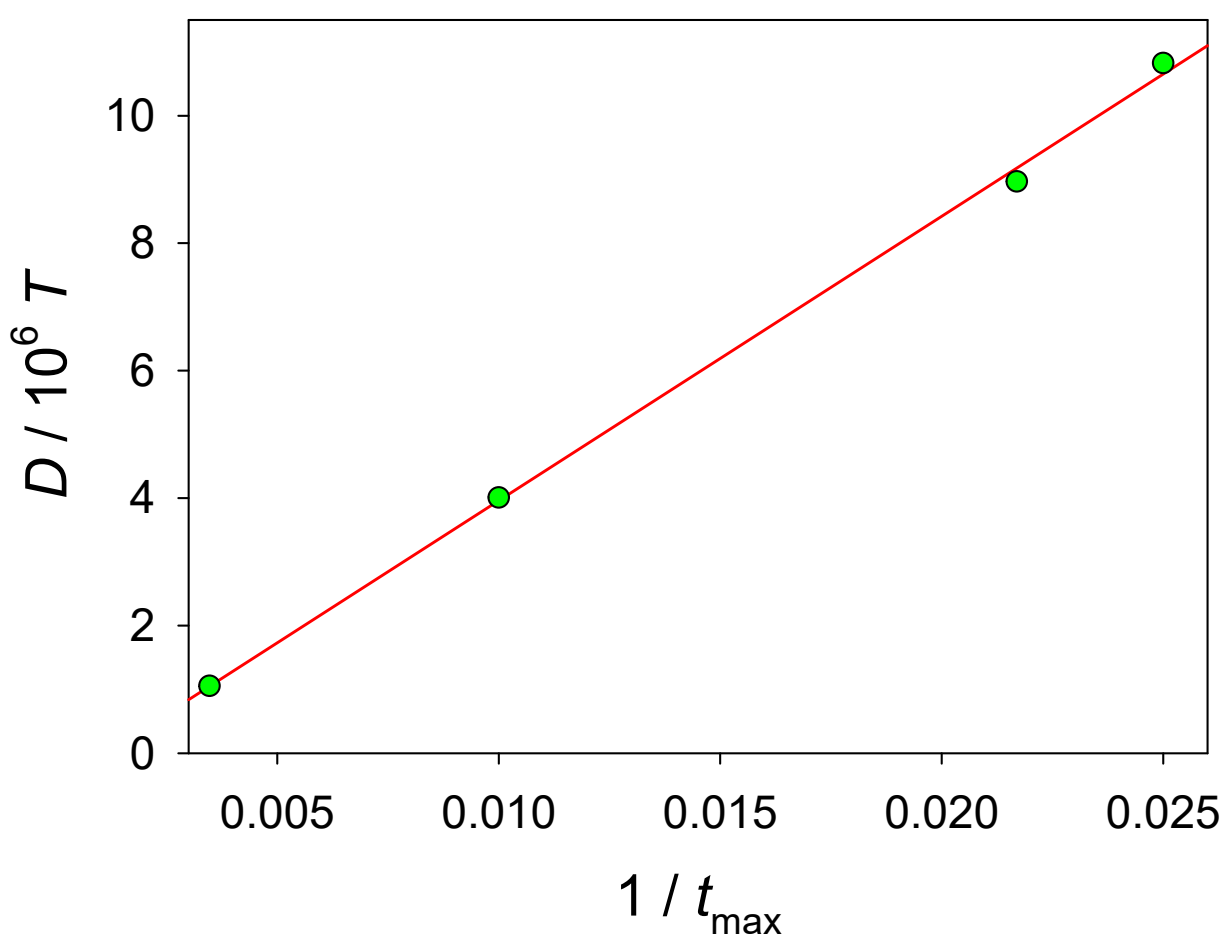

Figure 13: Self-diffusion coefficient $D$ divided by temperature $T$ as a function of inverse activation time of diffusion $t_{\max }$. 


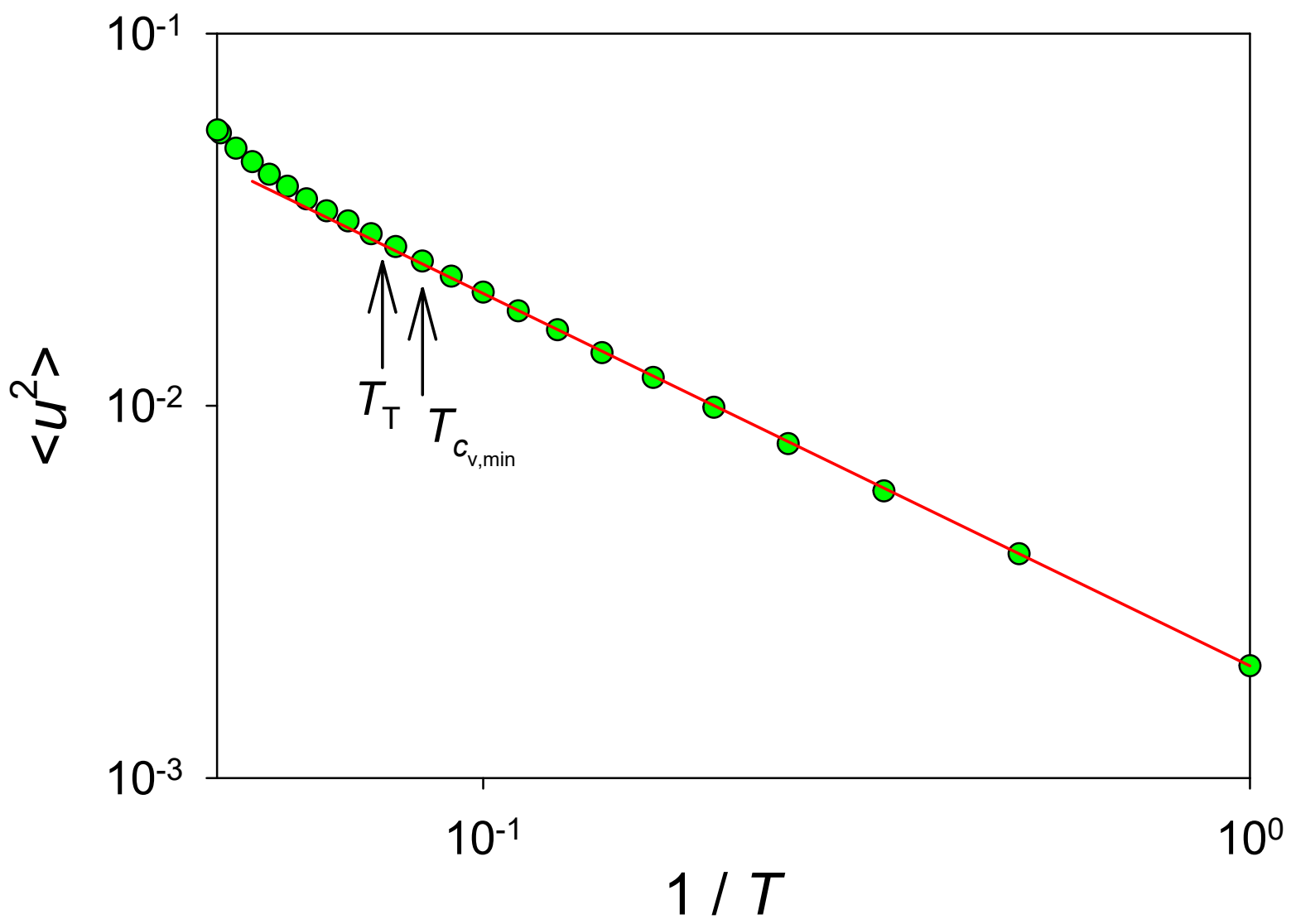

Figure 14: Debye-Waller factor $\left\langle u^{2}\right\rangle$ as a function of inverse temperature $1 / T$. The red line represents a power-law fit for $T<T_{\mathrm{T}}$. The Tammann temperature $T_{\mathrm{T}} \approx 13.5$ is marked by an arrow. $T_{c_{V, \text { min }}} \approx 12$ marks the crossover temperature of the $c_{V, \text { min }}$ line at $\rho=1.8$.

can be calculated at least for high temperatures close to the LS. Fig. 13 shows that $D / T$ scales linearly with inverse $t_{\max }$.

Another interesting quantity that can be estimated directly from the MSD is the Debye-Waller factor (DWF) $\left\langle u^{2}\right\rangle$, measuring oscillations of atoms around their equilibrium lattice positions. Starr et al. [33] and Larini et al. [34] defined the DWF as the MSD after the crossover from the ballistic to the vibrational regime. Following this definition, the DWF was calculated and is shown in Fig. 14 as a function of inverse temperature in a double logarithmic plot. Raising the temperature causes an increase of the DWF, which can be described by a power-law fit. For temperatures $T>T_{\mathrm{T}}$, an accelerated 
increase of the DWF can be observed, deviating from the power-law. This is an interesting result, since the behavior of the DWF reflects different dynamic regimes. When the Tammann temperature $T_{\mathrm{T}}$ is exceeded, increasing oscillation amplitudes grow faster than the power-law, allowing the atoms to diffuse. Obversely, the power-law behavior of the DWF indicates purely vibrational motion. In addition, a comparison of the temperature dependence of the DWF and that of the isochoric heat capacity $c_{V}$ is of interest. As discussed in the supplemental material (cf. Fig. S2), the line of the minimum $c_{V \text {,min }}$ bounds a phase region with anomalous thermodynamic behavior, characterizing premelting. The Tammann temperature of $T_{\mathrm{T}} \approx 13.5$ roughly corresponds to the intersection of the $c_{V \text {,min }}$ curve with the isochore $\rho=1.8$ at $T \approx 12$ (cf. Fig. 14). This indicates a close connection between the occurrence of anomalous thermodynamic behavior, reported in Ref. [13], and the occurrence of bulk mobility in the stable fcc-solid.

\subsection{Vibrational density of states}

A quantity describing the distribution of vibrational frequencies of an atomic system is given by the vibrational density of states (VDOS). The $\operatorname{VDOS} \rho_{\mathrm{V}}(\omega)$ can be calculated from the normalized velocity autocorrelation function $(\mathrm{VACF}) \psi(t)=\langle\boldsymbol{v}(t) \boldsymbol{v}(0)\rangle /\left\langle\boldsymbol{v}(0)^{2}\right\rangle$ using the Fourier transformation

$$
\rho_{\mathrm{V}}(\omega)=2 \int_{0}^{\infty} \psi(t) \cos (\omega t) d t
$$

with the frequency $\omega$. Near the S/F transition, a typical behavior of both phases should clearly emerge in $\psi(t)$ and $\rho_{\mathrm{V}}(\omega)$. VACF and VDOS were calculated for temperatures in a range from $T=19$ to 26 . The VACF was sampled over a span of 15000 time steps, where its decay is sufficient to execute the integral in Eq. (6).

Fig. 15.a depicts the normalized VACF. As expected, backscattering of atoms is much 

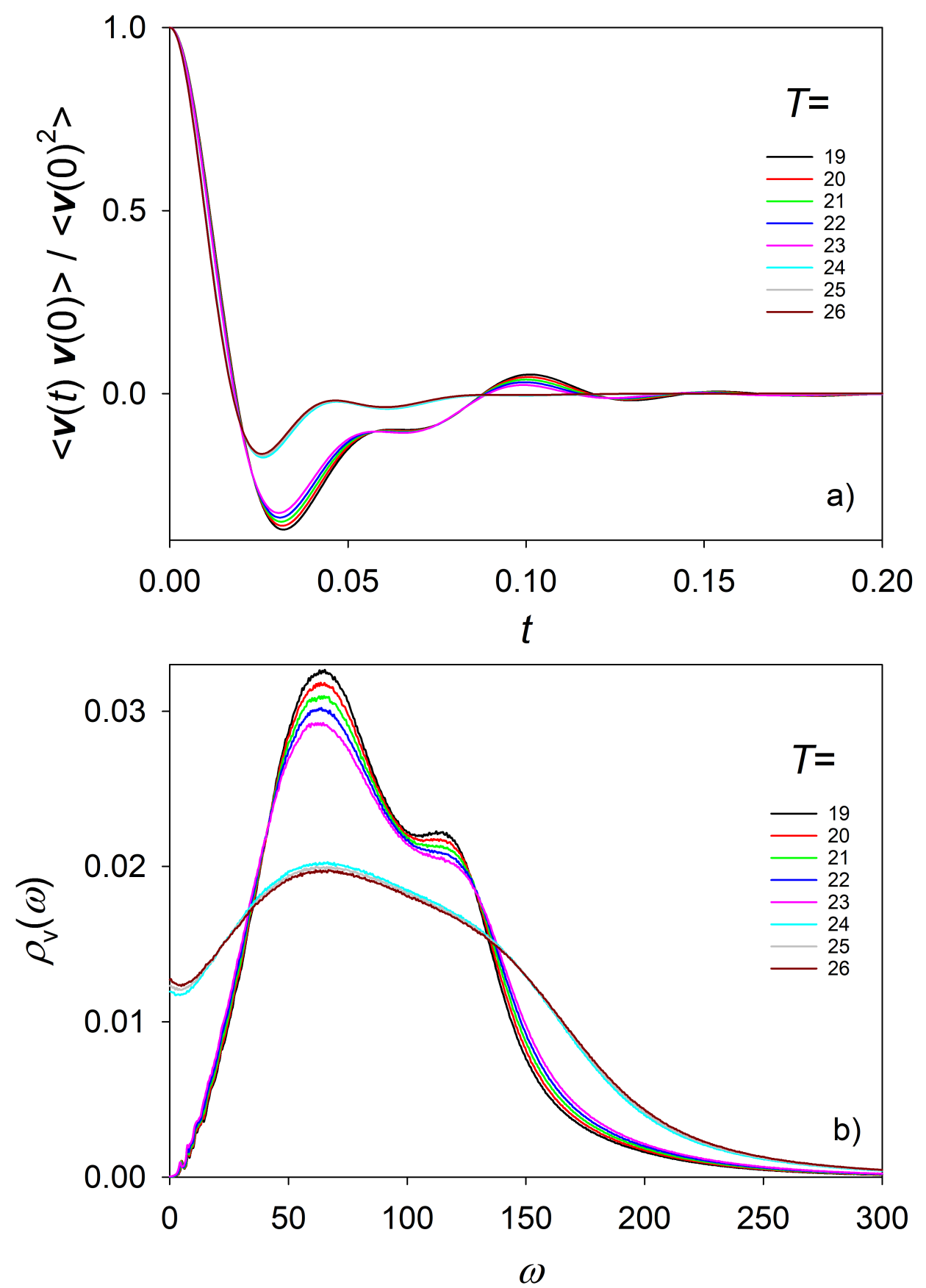

Figure 15: a) Normalized velocity autocorrelation function and b) vibrational density of states along the isochore $\rho=1.8$ for $T=19$ to 26 . 
more pronounced in the solid state and the transition to the fluid state can be clearly seen at a temperature of $T=24$. A corresponding behavior was observed for the VDOS as shown in Fig. 15.b. The position of the main solid state peak shifts slightly towards lower frequencies upon heating, an effect known as softening a solid. The magnitude of this peak decreases with rising temperature. A remarkable jump can be observed at $T=23$, widening the vibrational peak of the fluid state. The difference between the solid and fluid phase is most evident at $\omega=0$, where $\rho_{\mathrm{V}}(0)$ is finite for a fluid, while $\rho_{\mathrm{V}}(0)=0$ for a solid. Since $\rho_{\mathrm{V}}(0)$ is related to diffusivity, a finite value indicates incipient diffusion.

Zhang et al. [12] examined the reduced $\operatorname{VDOS} \rho_{\mathrm{V}}(\omega) / \omega^{2}$ [35] of a superheated $\mathrm{Ni}$ crystal and found a boson peak at low $\omega$, whose occurrence was interpreted in terms of collective atomic rearrangement motions. Regardless whether the low-frequency behavior appears in the sense of a boson-like peak, an investigation of the reduced VDOS of the superheated LJ fcc-crystal is of general interest, as discussed in the supplemental material.

\section{Conclusions}

Homogeneous melting is often accompanied by an anomalous behavior of thermodynamic properties [13], frequently suggested as an indicator for premelting. A question arising in this context is: To what extent can corresponding effects be found in the structure and dynamics of a crystal? In the present study, this issue was analyzed for the LJ fcc-solid with molecular dynamics simulations. In order to specify the region of interest, the limit of superheating as an upper bound of the metastable solid state and the melting point were estimated with the $\mathrm{Z}$ method. If melting precursors become apparent in a solid, a weakening of the crystalline order has to be expected. Therefore, translational order was investigated by means of the number of nearest neighbors and a translational order metric based on the PDF. Strong restructuring effects were found especially in the first 
and second shell of the PDF, when approaching the S/F transition. The first peak of the PDF moves to smaller interatomic distances and the number of first nearest neighbors is reduced. The second shell of the PDF already loses its translation order at about $T \approx 0.5 T_{\mathrm{m}}$, resulting in a broad distribution of atoms in this shell. Both effects favor the formation of thermal vacancies [29], which in turn allow for an activation of self-diffusion in the crystal. Investigations of the mean-squared displacement showed that diffusion processes are activated at the Tammann temperature $T_{\mathrm{T}} \approx 2 T_{\mathrm{m}} / 3$. An analysis of the non-Gaussian parameter revealed a remarkable peak when approaching $T_{\mathrm{m}}$. This indicates strong heterogeneous dynamics, which is generally attributed to the appearance of stringlike collective atomic motion within the crystal. In addition, a changing temperature dependence of the Debye-Waller factor at $T_{\mathrm{T}}$ supports the findings of increasing mobility in the fcc-solid. Furthermore, a pronounced peak was detected in the vibrational density of states at low frequencies. In summary, melting precursors are present in the structure and dynamics of the LJ fcc-solid and thus reflect the anomalous behavior of thermodynamic properties in a large portion of the approach to the melting point.

\section{Acknowledgments}

Computational support was given by the High Performance Computing Center Stuttgart (HLRS) under the grant MMHBF2. Furthermore, we gratefully acknowledge the Paderborn Center for Parallel Computing (PC2) for the generous allocation of computer time on the OCuLUS and Noctua cluster. We thank Gabriela Guevara-Carrion for her valuable support.

\section{DATA AVAILABILITY}

The data that support the findings of this study are available from the corresponding author upon reasonable request. 


\section{Supplemental material}

The supplemental material contains a brief review of the thermodynamic results obtained by Köster et al. [13] as well as additional information and tests to complement the main body of this work. The FP was determined on the basis of the Hansen-Verlet criterion and was additionally checked by the Raveché-Mountain-Streett criterion. Furthermore, the VDOS at low frequencies is discussed.

\section{References}

[1] F. A. Lindemann, "Über die Berechnung molekularer Eigenfrequenzen," Phys. Z. 11, 609 (1919).

[2] M. Born, "Thermodynamics of Crystals and Melting," J. Chem. Phys. 7, 591 (1939).

[3] Z. H. Jin, P. Gumbsch, K. Lu, and E. Ma, "Melting mechanisms at the limit of superheating," Phys. Rev. Lett. 87, 055703 (2001).

[4] H. J. Fecht and W. L. Johnson, "Entropy and enthalpy catastrophe as a stability limit for crystalline material," Nature 334, 50 (1988).

[5] W. Kauzmann, "The nature of the glassy state and the behavior of liquids at low temperatures," Chem. Rev. 43, 219 (1948).

[6] L. Burakovsky, D. L. Preston, and R. Silbar, "Melting as a dislocation-mediated phase transition," Phys. Rev. B 61, 15011 (2000).

[7] L. Gomez, A. Dobry, Ch. Geuting, H. T. Diep, and L. Burakovsky, "Dislocation lines as the precursor of the melting of crystalline solids observed in Monte Carlo simulations," Phys. Rev. Lett. 90, 095701 (2003).

[8] F. Delogu, "Cooperative dynamics and self-diffusion in superheated crystals," J. Phys. Chem. B 109, 15291 (2005).

[9] F. Delogu, "Cooperative atomic displacements and melting at the limit of superheating," J. Phys. Chem. B 110, 3281 (2006).

[10] A. B. Belonoshko, S. Davis, N. V. Skorodumova, P. H. Lundow, A. Rosengren, and B. Johansson, "Properties of the fcc Lennard-Jones crystal model at the limit of superheating," Phys. Rev. B 76, 064121 (2007).

[11] X.-M. Bai and M. Li , "Ring-diffusion mediated homogeneous melting in the superheating regime," Phys. Rev. B 77, 134109 (2008).

[12] H. Zhang, M. Khalkhali, Q. Liu, and J. F. Douglas, "String-like cooperative motion in homogeneous melting," J. Chem. Phys. 138, 12A538 (2013).

[13] A. Köster, P. Mausbach, and J. Vrabec, "Premelting, solid-fluid equilibria, and thermodynamic properties in the high density region based on the Lennard-Jones potential," J. Chem. Phys. 147, 144502 (2017). 
[14] A. B. Belonoshko, N. V. Skorodumova, A. Rosengren, and B. Johansson, "Melting and critical superheating," Phys. Rev. B 73, 012201 (2006).

[15] F. González-Cataldo, S. Davis, and G. Gutiérrez, "Z method calculations to determine the melting curve of silica at high pressures," J. Phys.: Conf. Ser. 720012032 (2016).

[16] K. Yin, X. Lu, H. Zhou, and Y. Sun, "Thermodynamic stability limit of the crystalline state from the Gibbs perspective," Phys. Rev. B 98, 144113 (2018).

[17] V. Olguín-Arias, S. Davis, and G. Gutiérrez, "Extended correlations in the critical superheated solid," J. Chem. Phys. 151, 064507 (2019).

[18] S. Deublein, B. Eckl, J. Stoll, S. V. Lishchuk, G. Guevara-Carrion, C. W. Glass, T. Merker, M. Bernreuther, H. Hasse, and J. Vrabec, "ms2: A molecular simulation tool for thermodynamic properties," Comp. Phys. Commun. 182, 2350-2367, (2011).

[19] C. W. Glass, S. Reiser, G. Rutkai, S. Deublein, A. Köster, G. Guevara-Carrion, A. Wafai, M. Horsch, M. Bernreuther, T. Windmann, H. Hasse, and J. Vrabec, "ms2: A molecular simulation tool for thermodynamic properties, new version release," Comp. Phys. Commun. 185, 3302-3306, (2014).

[20] G. Rutkai, A. Köster, G. Guevara-Carriona, T. Janzen, M. Schappals, C.W. Glass, M. Bernreuther, A. Wafai, S. Stephan, M. Kohns, S. Reiser, S. Deublein, M. Horsch, H. Hasse, and J. Vrabec, "ms2: A molecular simulation tool for thermodynamic properties, release 3.0," Comp. Phys. Commun. 221 , 343-351 (2017).

[21] A. M. Nieves and T. Sinnoa, "An enthalpy landscape view of homogeneous melting in crystals," J. Chem. Phys. 135, 074504 (2011).

[22] X.-M. Bai and M. Li, "Differences between solid superheating and liquid supercooling," J. Chem. Phys. 123, 151102 (2005).

[23] M. C. Abramo, C. Caccamo, D. Costa, P. V. Giaquinta, G. Malescio, G. Munaò, and S. Prestipino, "On the determination of phase boundaries via thermodynamic integration across coexistence regions," J. Chem. Phys. 142, 214502 (2015).

[24] J.-P. Hansen and L. Verlet, "Phase Transitions of the Lennard-Jones System," Phys. Rev. 184, 151 (1969).

[25] M. Gottschalk, "An EOS for the Lennard-Jones fluid: A virial expansion approach," AIP Adv. 9, 125206 (2019).

[26] A. Ahmed and R. J. Sadus, "Solid-liquid equilibria and triple points of n-6 LennardJones fluids," J. Chem. Phys. 131, 174504 (2009); Erratum 133, 229902 (2010).

[27] A. J. Schultz and D. A. Kofke, "Comprehensive high-precision high-accuracy equation of state and coexistence properties for classical Lennard-Jones crystals and lowtemperature fluid phases," J. Chem. Phys. 149, 204508 (2018).

[28] J. R. Errington, P. G. Debenedetti, and S. Torquato, "Quantification of order in the Lennard-Jones system," J. Chem. Phys. 118, 2256 (2003).

[29] M. J. Pozo, S. Davis, and J. Peralta, "Statistical distribution of thermal vacancies close to the melting point," Physica B 457, 310 (2015).

[30] G. Tammann, "Die Temperatur des Beginns innerer Diffusion in Kristallen," Z. Anorg. Allg. Chem. 157, 321 (1926). 
[31] G. Tammann ,"Lehrbuch der Metallkunde. Chemie und Physik der Metalle und ihrer Legierungen," 4. erweiterte Auflage, Leopold Voß, Leipzig (1932).

[32] H. Zhang, X. Wang, A. Chremos, and J. F. Douglas "Superionic $\mathrm{UO}_{2}$ : A model anharmonic crystalline material," J. Chem. Phys. 150, 174506 (2019).

[33] F. W. Starr, S. Sastry, J. F. Douglas, and S. C. Glotzer, "What do we learn from the local geometry of glass-forming liquids?," Phys. Rev. Lett. 89, 125501 (2002).

[34] L. Larini, A. Ottochian, C. De Michele, and D. Leporini, "Universal scaling between structural relaxation and vibrational dynamics in glass-forming liquids and polymers," Nat. Phys. 4, 42 (2008).

[35] T. S. Grigera, V. Martin-Mayor, G. Parisi, and P. Verrocchio, "Phonon interpretation of the 'boson peak' in supercooled liquids," Nature 422, 289 (2003). 


\title{
Supplemental Material to:
}

\section{Structure and dynamics of the Lennard-Jones fcc-solid focusing on melting precursors}

\author{
Peter Mausbach ${ }^{\mathrm{a}}$, Robin Fingerhut ${ }^{\mathrm{b}}$, and Jadran Vrabec $^{\mathrm{b}, *}$ \\ ${ }^{a}$ Plant and Process Engineering, Technical University of Cologne, 50678 Cologne, Germany \\ ${ }^{\mathrm{b}}$ Thermodynamics and Process Engineering, Technical University Berlin, 10587 Berlin, \\ Germany \\ ${ }^{*}$ Corresponding author, E-mail address: vrabec@tu-berlin.de
}

This supplementary material contains a brief review of the current status of thermodynamic results obtained from Köster et al. [1] as well as additional information and tests to complement the main text. 


\section{Review on thermodynamic anomalies near melting}

Köster et al. [1] determined the entire set of thermodynamic properties of the LennardJones fcc-solid over a large phase region. Close to the solid-fluid (S/F) phase transition, several properties showed an accelerated anomalous behavior. In this section, this issue is reviewed exemplarily for the isochoric heat capacity $c_{V}$.

Fig. 1.a depicts density dependent $c_{V}$ data from simulations of Köster et al. [1] along three isotherms $T=1.3,6$ and 22 in comparison with results from an EOS developed by Schultz and Kofke [2], supplemented by an EOS taking vacancy effects into account. In general, $c_{V}$ values of both studies $[1,2]$ show a good agreement in the solid region. This is a challenging comparison since the isochoric heat capacity is a second order derivative of the Helmholtz energy so that the consistency of the $c_{V}$ data of Köster et al. [1] can be deduced.

For the temperature $T=1.3$, the freezing point $(\mathrm{FP})$ and melting point $(\mathrm{MP})$ coordinates of Refs. $[1,2]$ are almost identical. Here, the isochoric heat capacity $c_{V}$ exhibits a pronounced minimum when approaching the MP, with a position roughly at the MP. Entering the metastable two-phase coexistence region by reducing the density causes an anomalous increase of $c_{V}$, followed by a sudden drop close to the FP which signals that the limit of superheating (LS) is reached. The simulation data of the fluid phase together with the metastable data exhibit a $\lambda$-like shape at the $\mathrm{S} / \mathrm{F}$ phase transition as discussed in Ref. [1]. Increasing the temperature to $T=6$ and 22 leads to quantitatively somewhat diverging coexistence lines of Refs. [1] and [2]. However, the minimum of $c_{V}$ shifts to the stable solid region, regardless of differing MP locations in Refs. [1] and [2].

A similar behavior can be observed for the temperature dependence of the isochoric heat capacity along the isochore $\rho=1.3$ as shown in Fig. 1.b. Therein, the temperature was scaled with the melting temperature $T_{\mathrm{m}}$ of Schultz and Kofke at $\rho=1.3$ to allow for a direct comparison. Note that the formula for the ML in Ref. [2] contains a misprint. 

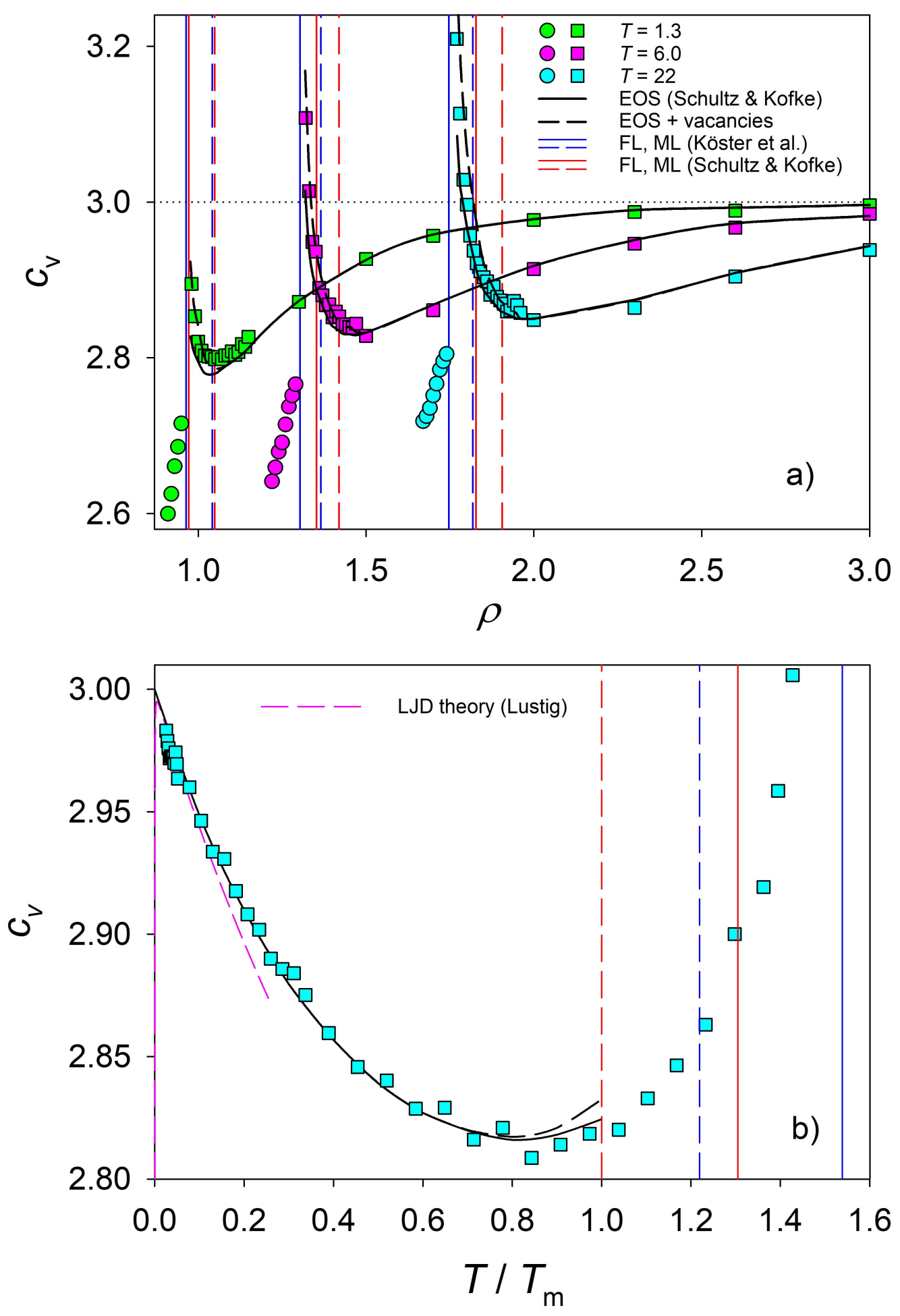

Figure S1: Isochoric heat capacity $c_{V}$, a) of the fluid and fcc solid phase along three isotherms $T=1.3,6$ and 22 and b) in the solid phase along the isochore $\rho=1.3$. Squares present simulation data of Köster et al. [1] in the solid, circles in the fluid phase. $c_{V}$ values obtained from the EOS of Schultz and Kofke [2] are shown as black solid lines, values from an EOS with additional consideration of vacancies are shown as black dashed lines. Vertical lines indicate the FP and the MP: blue - Köster et al. [1], red - Schultz and Kofke [2]. Vertical dashed lines indicate the MP, vertical solid lines indicate the FP. The dotted line in a) indicates the Dulong-Petit value $c_{\mathrm{V}}=3$. Additionally shown in $\mathrm{b}$ ) is $c_{\mathrm{V}}$ for small $T$ according to the LJD theory of Lustig [3]. All temperatures in b) are reduced by the melting temperature $T_{\mathrm{m}}$ of Schultz and Kofke at $\rho=1.3$. 
The pre-factor for $\rho_{m e l t}^{f c c}$, Eq. (2), Table I, should be $\beta^{-1 / 4}$ instead of $\beta^{-1 / 2}$ [4]. Again, both studies show a good agreement. An accelerated increase of $c_{V}$ can be observed within the two-phase coexistence region, however, the onset of the anomalous increase of $c_{V}$ starts below the melting temperature at $T \approx 0.8 T_{\mathrm{m}}$. Such a behavior has frequently been suggested as an indicator for premelting.

The minimum $c_{V, \text { min }}$ opens a possibility to narrow down the phase region with premelting effects. In Fig. S2, the course of the minimum $c_{V, \text { min }}$ at constant density estimated from the simulation data of Köster et al. [1] is shown. A remarkable premelting zone is visible within the stable solid region. Fig. S2 additionally shows the courses of the FL of Gottschalk [5] as well as Ahmed and Sadus [6] which are almost identical with the FL of Köster et al. [1]. The MP and LS (both with the Z method, cf. section 3 main-text) as well as the FP (with the Hansen-Verlet criterion, cf. section 4) are depicted by symbols in Fig. S2. 


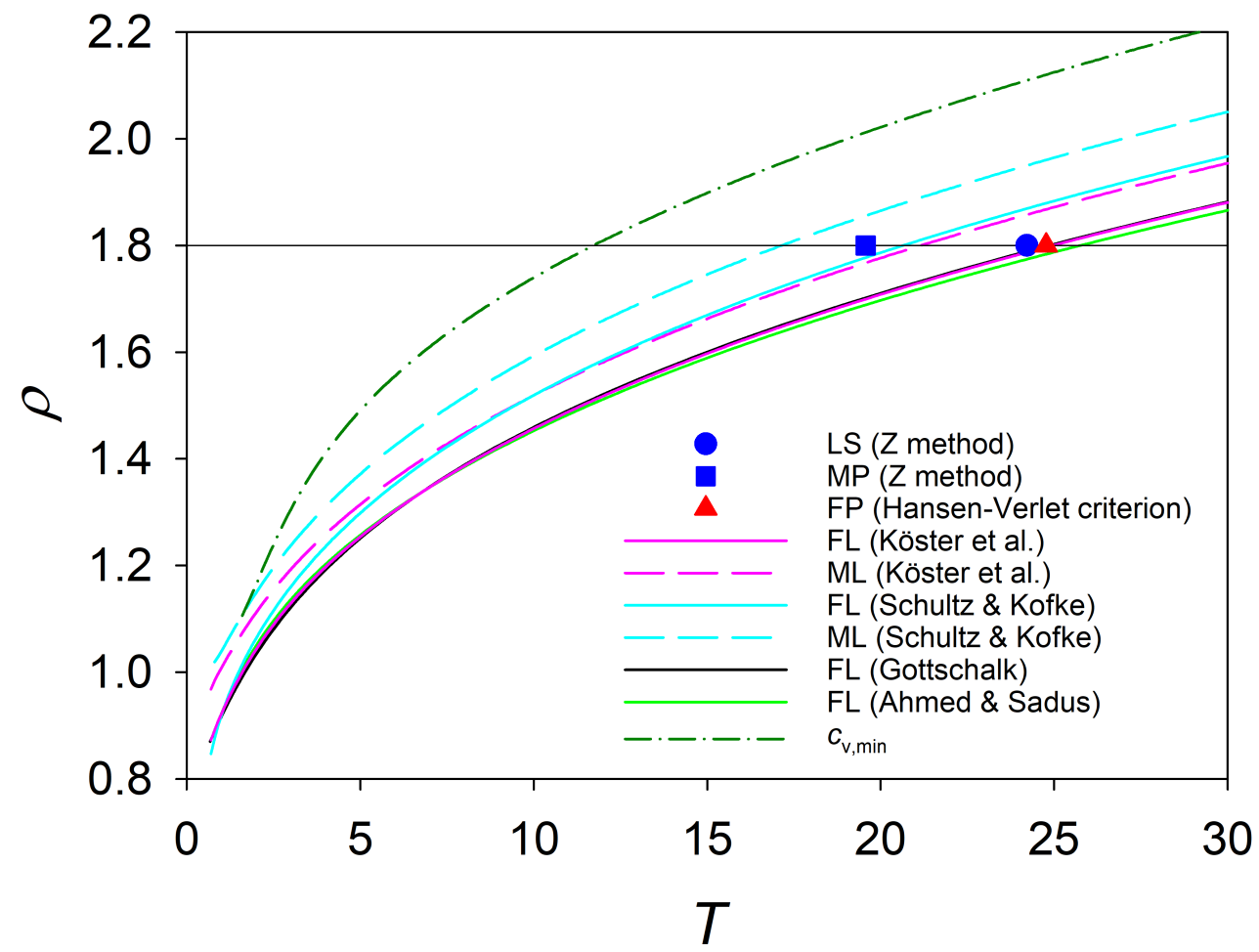

Figure S2: Density $\rho$ as a function of temperature $T$ along the freezing (FL) and melting lines (ML) of Köster et al. [1] and Schultz and Kofke [2]. The FL by Gottschalk [5] and Ahmed and Sadus [6] is shown as well. Furthermore, the dash-dotted line indicates the minimum $c_{\mathrm{V} \text {,min }}$ at constant density estimated from the simulation data of Köster et al. [1]. The MP and LS, calculated with the $\mathrm{Z}$ method, and the FP, calculated with the Hansen-Verlet criterion for $N=10976$, are shown by symbols. 


\section{Molecular simulation parameters}

Table S1 compiles the simulation parameters used in this study.

Table S1: Simulation parameters for the Z method and functions sampled in this study. Function length, equilibration and production period are given in time steps $\Delta t$.

\begin{tabular}{c||c|c|c|c|c|c|c}
\hline $\begin{array}{c}\text { Method/ } \\
\text { Function }\end{array}$ & Ensemble & $N$ & $r_{c}$ & $\Delta t$ & $\begin{array}{c}\text { Function } \\
\text { length }\end{array}$ & $\begin{array}{c}\text { Equilibration } \\
\text { steps }\end{array}$ & $\begin{array}{c}\text { Production } \\
\text { steps }\end{array}$ \\
\hline Z & NVE & 256 & 2.6 & 0.000225 & & & $10^{6}$ \\
& & 500 & 3.2 & & & & $10^{6}$ \\
& & 1372 & 4.5 & & & & $3.2 \times 10^{5}$ \\
& & 4000 & 6.0 & & & & $5 \times 10^{5}$ \\
& & 10976 & 6.5 & & & & $3.2 \times 10^{5}$ \\
& & 32000 & 6.5 & & & & $5 \times 10^{5}$ \\
& & 108000 & 6.5 & & & & $10^{6}$ \\
\hline PDF & NVT & 10976 & 9.1 & 0.001 & & $2 \times 10^{5}$ & $10^{7}$ \\
\hline MSD & NVT & 10976 & 9.1 & 0.001 & $10^{6}-10^{7}$ & $2 \times 10^{5}$ & $1-2 \times 10^{7}$ \\
\hline VACF & NVT & 10976 & 9.1 & 0.0005 & 15000 & $5 \times 10^{5}$ & $8.5 \times 10^{5}$ \\
\hline
\end{tabular}




\section{$3 \quad$ Finite size effects of the $\mathrm{Z}$ method}

Fig. S3 depicts $(u, T)$ curves obtained from the $\mathrm{Z}$ method for different atom numbers $N$.

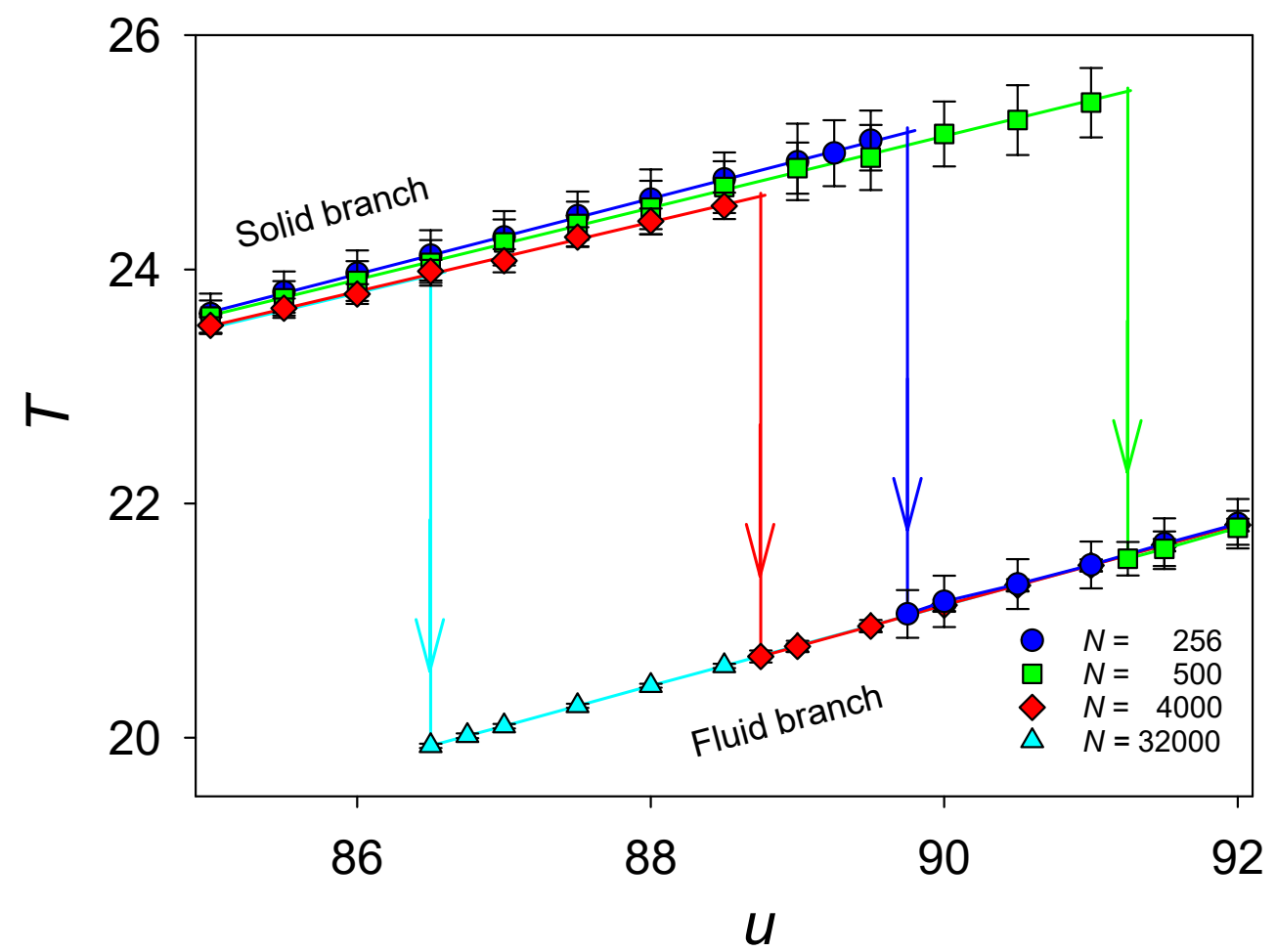

Figure S3: Temperature $T$ as a function of total energy $u$ obtained from microcanonical simulations with the $\mathrm{Z}$ method. Results for different atom numbers $N=256,500,4000$ and 32000 are shown along the isochore $\rho=1.8$. Vertical lines indicate the temperature drop from the LS to the MP. 


\section{$4 \quad$ Phenomenological freezing criteria}

Several approximate approaches based on structural information have been proposed to locate the freezing point (FP) coordinates. Perhaps the most successful phenomenological criterion for determining the freezing transition was introduced by Hansen and Verlet [7] on the basis of the static structure factor $S(q)$ of a uniform system. It is defined by the Fourier transformation

$$
S(q)=1+4 \pi \rho \int_{0}^{\infty}(g(r)-1) \frac{\sin (q r)}{q r} r^{2} d r
$$

where $q$ is the modulus of the wave vector $\boldsymbol{q}$. Hansen and Verlet noticed that the magnitude of the first peak of the fluid structure factor $S(q)$ is nearly 2.85 at the FP, which seems to be a universal feature that has been verified for many systems. Fig. S4 shows the amplitude of the first peak of $S(q)$ in the temperature range from $T=24$ to 26 . The maximum of the first peak exceeds the value 2.85 roughly at $T=24.75$. Fig. S5 depicts the temperature dependence of the intersection with 2.85 at $T=24.775$, which is close to the freezing temperature $T_{f}=24.972$ according to Köster et al. [1].

Another approximate rule for locating freezing is the Raveché-Mountain-Streett criterion [8], where the Raveché parameter of $R=g_{\min } / g_{\max }=0.2 \pm 0.02$ indicates freezing. $R$ is the ratio of the first non-zero minimum magnitude of $g(r)$ to the the first maximum magnitude of $g(r)$. The temperature dependence of the inverted Raveché parameter $R^{-1}$ is shown in Fig. S6, exhibiting the value $R^{-1}=5$ almost exactly on the FP according to the Hansen-Verlet criterion. 


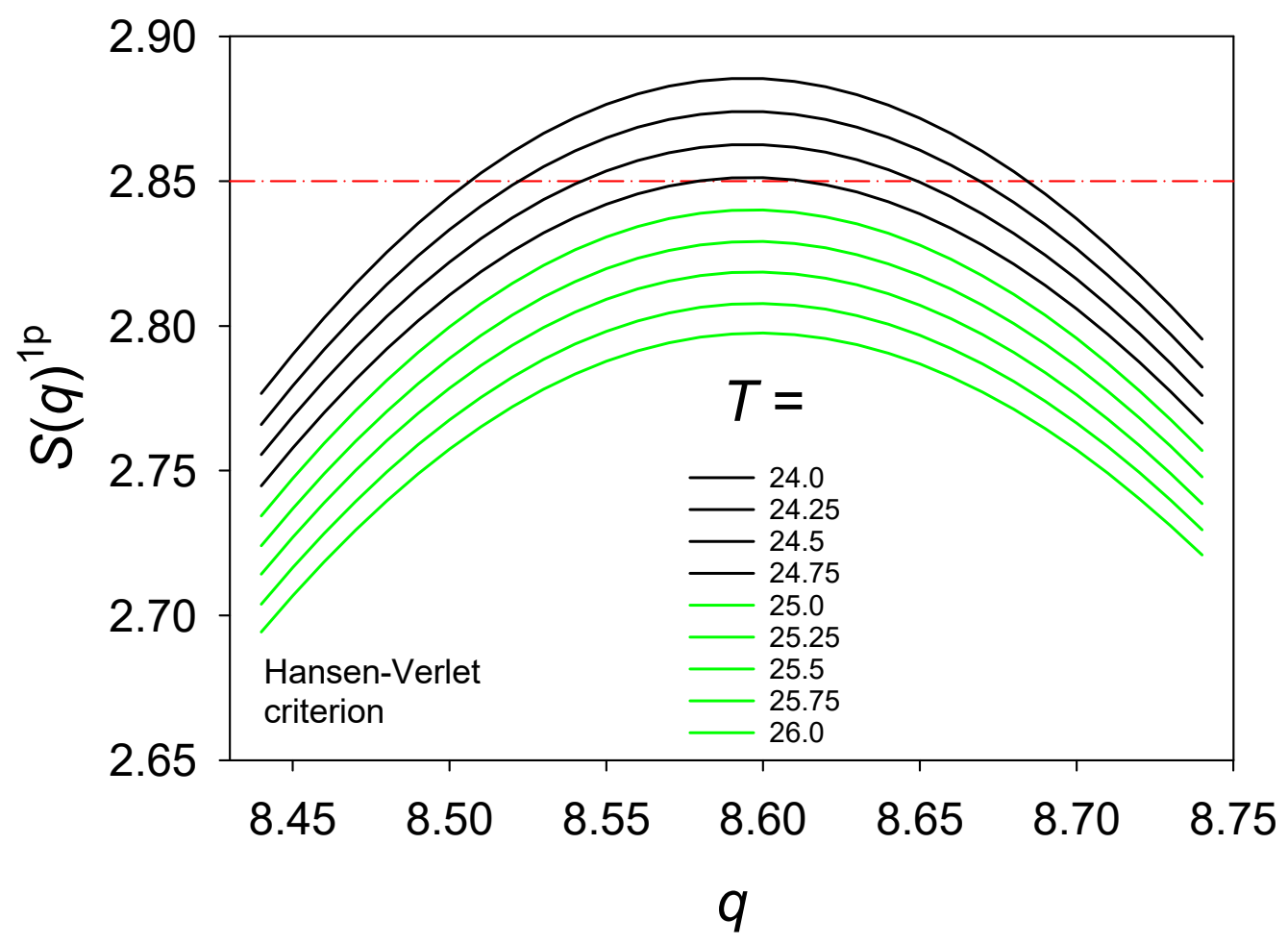

Figure S4: Temperature dependence of the magnitude of the first peak of $S(q)$ along the isochore $\rho=1.8$ for $T=24$ to 26 . Green curves represent the fluid region, black curves the two-phase coexistence region. The red, dash-dotted line marks the Hansen-Verlet criterion. 


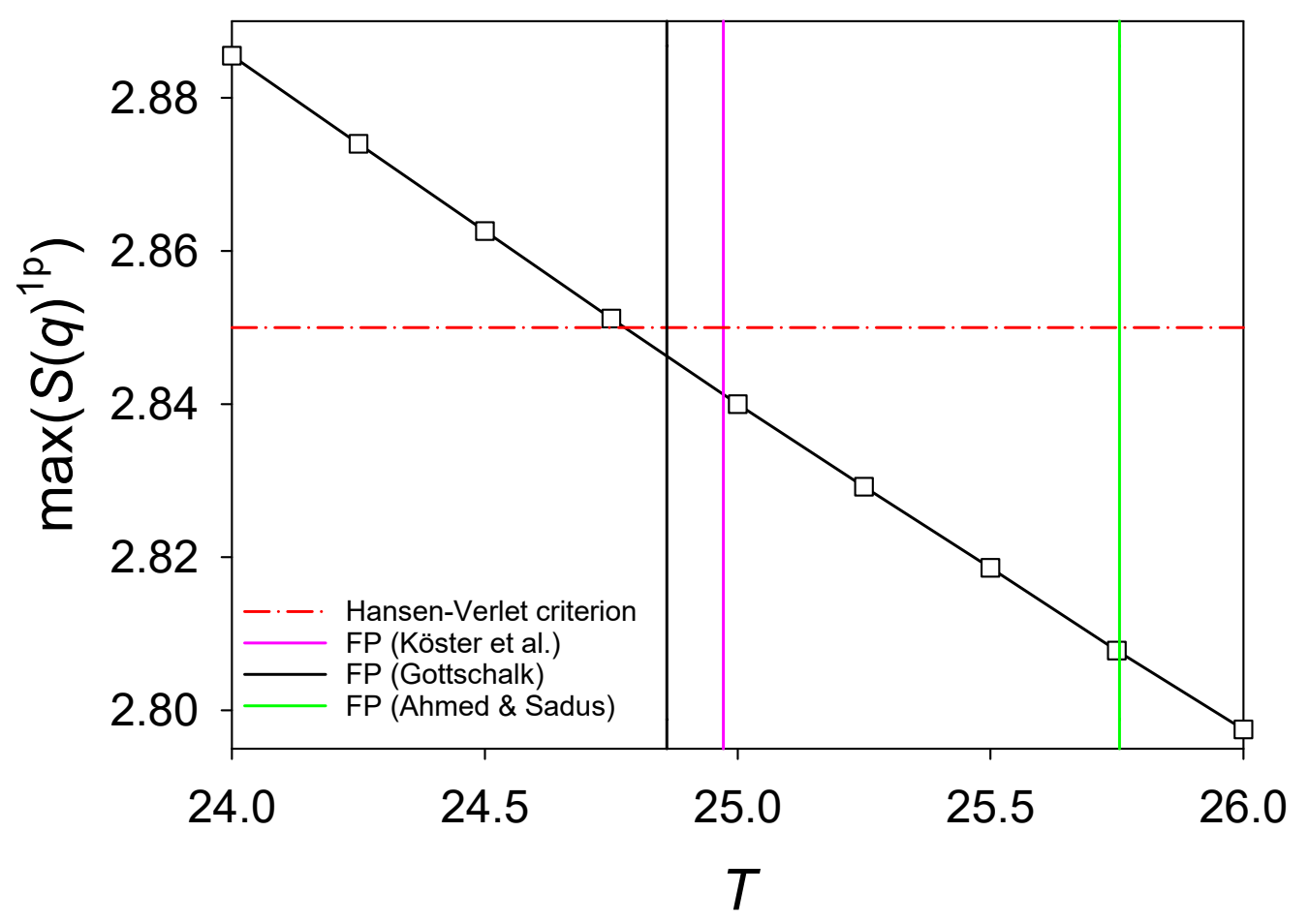

Figure S5: Temperature dependence of the magnitude of the first peak of $S(q)$ depicted by squares. The vertical lines represent the FP according to Köster et al. [1] (pink), Gottschalk [5] (black) as well as Ahmed and Sadus [6] (green). The red, dash-dotted line marks the Hansen-Verlet criterion. 


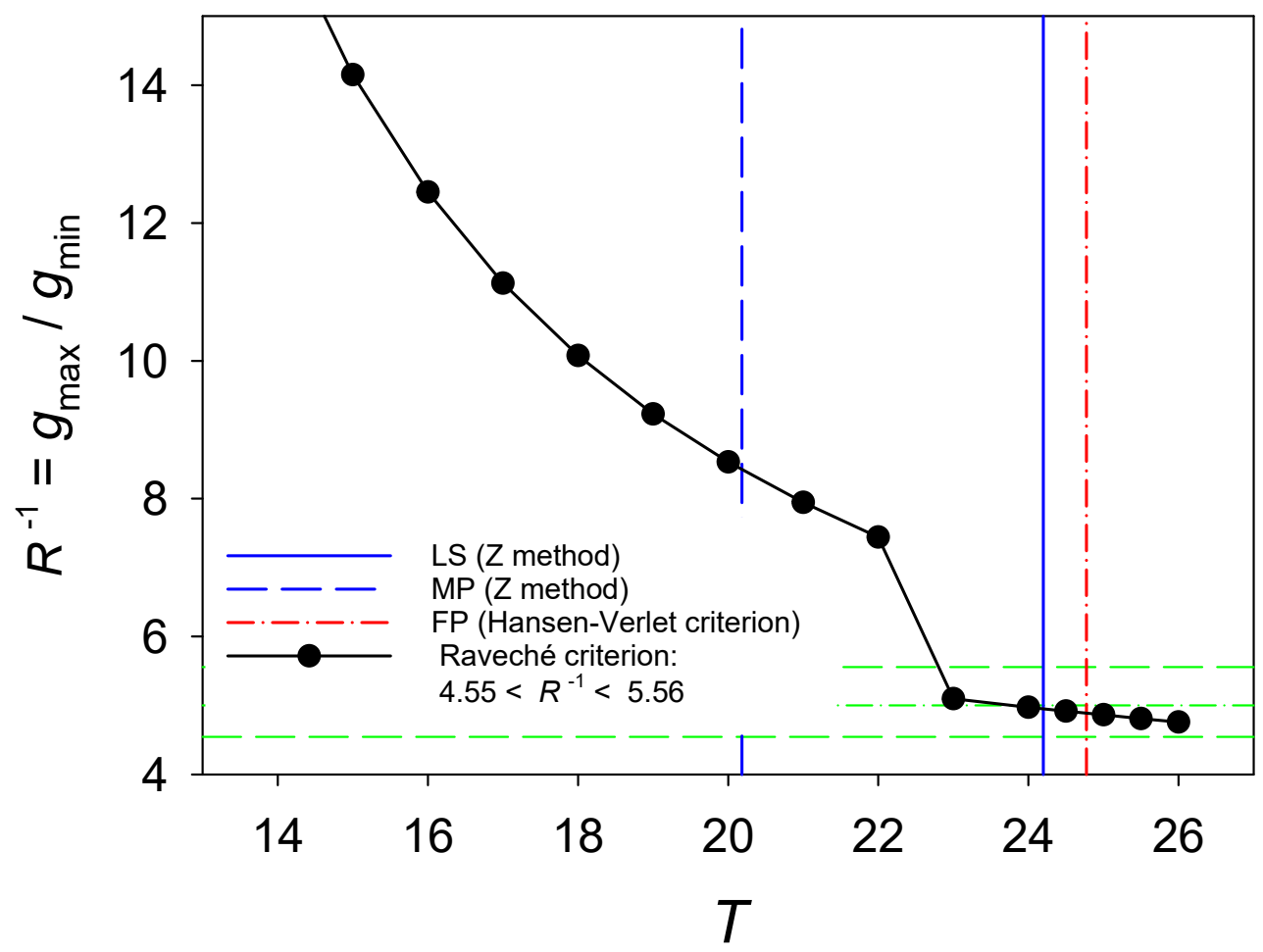

Figure S6: Temperature dependence of the inverse Raveché parameter $R^{-1}$. The horizontal dash-dotted line corresponds to $R=0.2$, dashed lines mark upper and lower bounds (all in green). The vertical dashed line marks the MP, the solid line the LS calculated with the $\mathrm{Z}$ method (both in blue). The vertical dashed dotted line (in red) marks the FP obtained from the Hansen-Verlet criterion. All data were generated with $N=10976$. 


\section{$5 \quad$ Running coordination number and nearest neigh- bors}

The running coordination number $n(r)$ is shown in Fig. S7 for selected temperatures between $T=1$ and 24 . The pronounced plateaus correspond to the minima $\left(r_{1, \min }, r_{2, \min }, \ldots\right)$ separating the peaks of the PDF shown in Fig. S8. The numbers on the right in Fig. S7 indicate the cumulated number of next nearest neighbors for a perfect fcc-crystal, i.e. $1 \mathrm{NN}=12,2 \mathrm{NN}=6,3 \mathrm{NN}=24, \ldots$

Fig. S9 depicts the temperature dependence of $3 \mathrm{NN}$ and $4 \mathrm{NN}$, where the numbers 3NN $=24$ and $4 \mathrm{NN}=12$ of a perfect fcc-crystal change quickly with increasing temperature. Jumps in the course of $3 \mathrm{NN}$ and $4 \mathrm{NN}$ indicate the solid/fluid transition. 


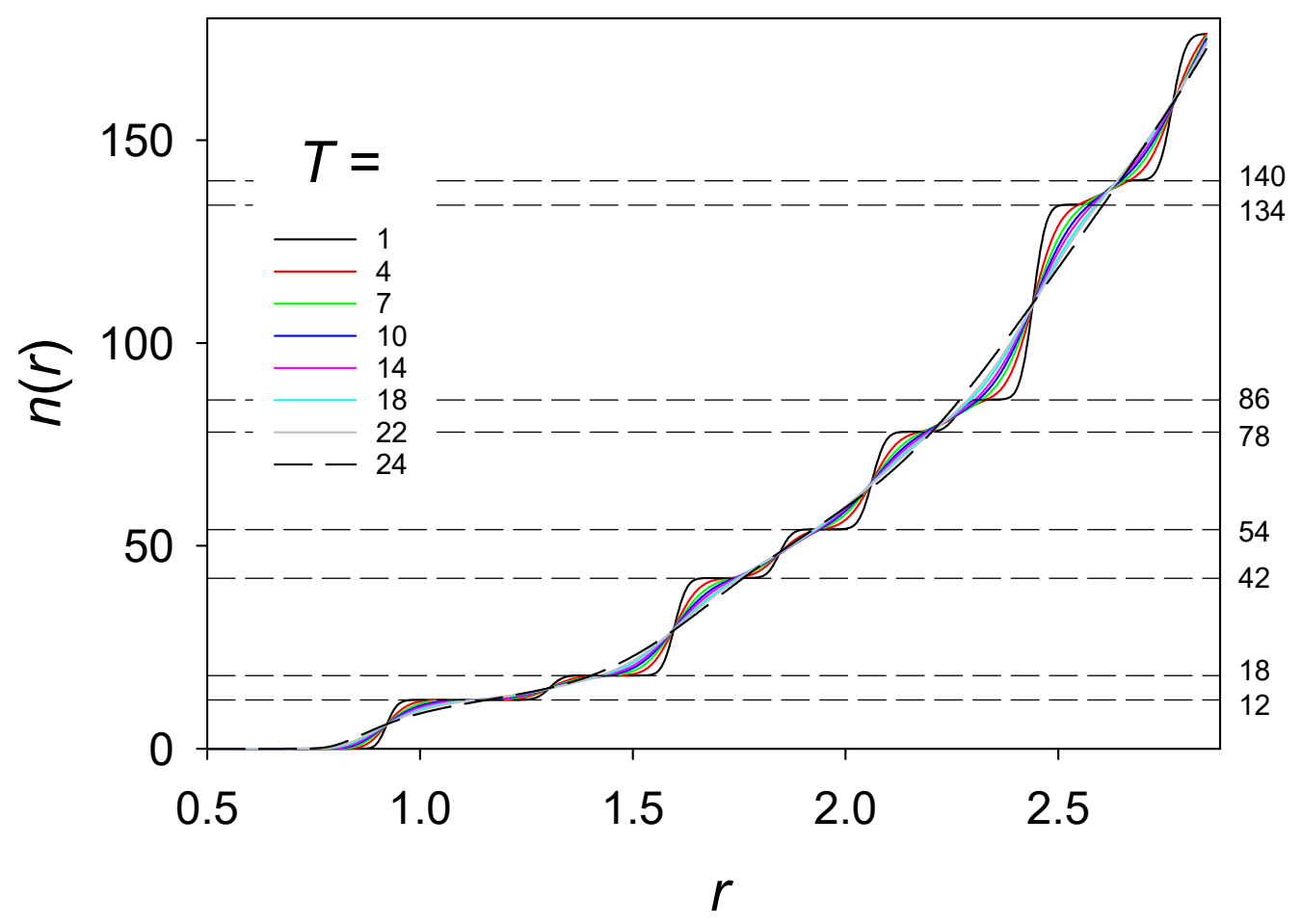

Figure S7: Running coordination number $n(r)$ along the isochore $\rho=1.8$ for $T=1$ to 24 . 


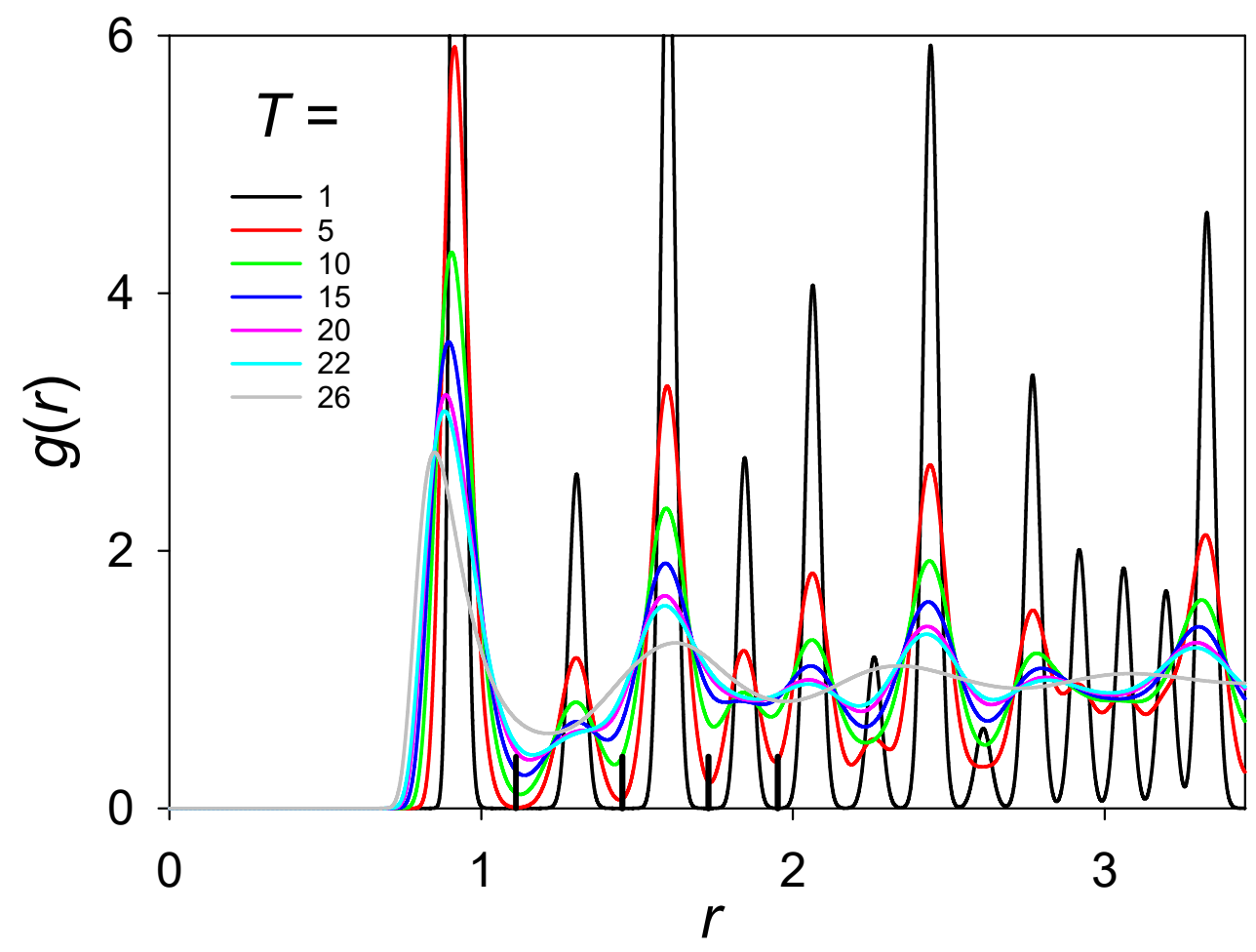

Figure S8: Pair distribution function $g(r)$ along the isochore $\rho=1.8$ for selected temperatures between $T=1$ and 26, depicted up to an atom-atom distance of $r \leq 3.45$. Bold lines on the lower horizontal axis mark the minima $\left(r_{1, \min }, r_{2, \min }, \ldots\right)$ separating the peaks of the PDF. 


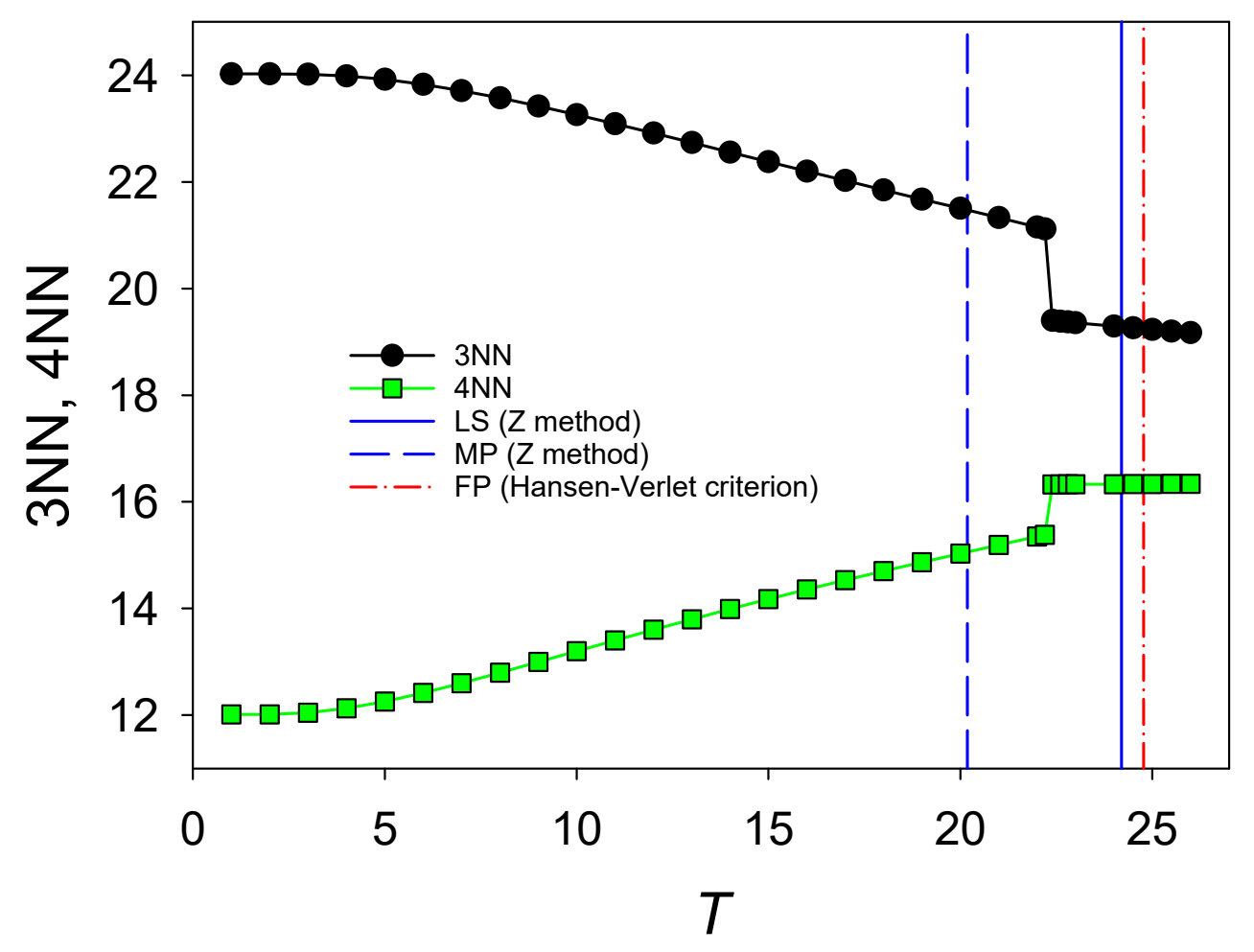

Figure S9: Temperature dependence of next nearest neighbors $3 \mathrm{NN}$ and $4 \mathrm{NN}$ along the isochore $\rho=1.8$ for $T=1$ to 26. Symbols and conditions are the same as in Fig. S6. 


\section{Diffusion mechanism in a perfect crystal}

As described in the accompanied paper, the main mechanism for the occurrence of diffusion in a perfect crystal are thermally activated jumps among short-time vacant equilibrium lattice sites [9]. A close inspection of the temperature dependence of $\Delta g(r, T)=$ $g(r, T)-g\left(r, T_{0}\right)$ may explain this. $\Delta g(r, T)$ with $T_{0}=1$ depicted in Fig. S10 shows that the interstitial space is occupied upon heating, although the long-range order is well visible as long as the sample remains in the solid state. This creates vacant lattice sites, at least in the short term [9]. The arrows mark rising temperature, with $\Delta g(r, T)$ increasingly resembling the curves of the fluid phase. 


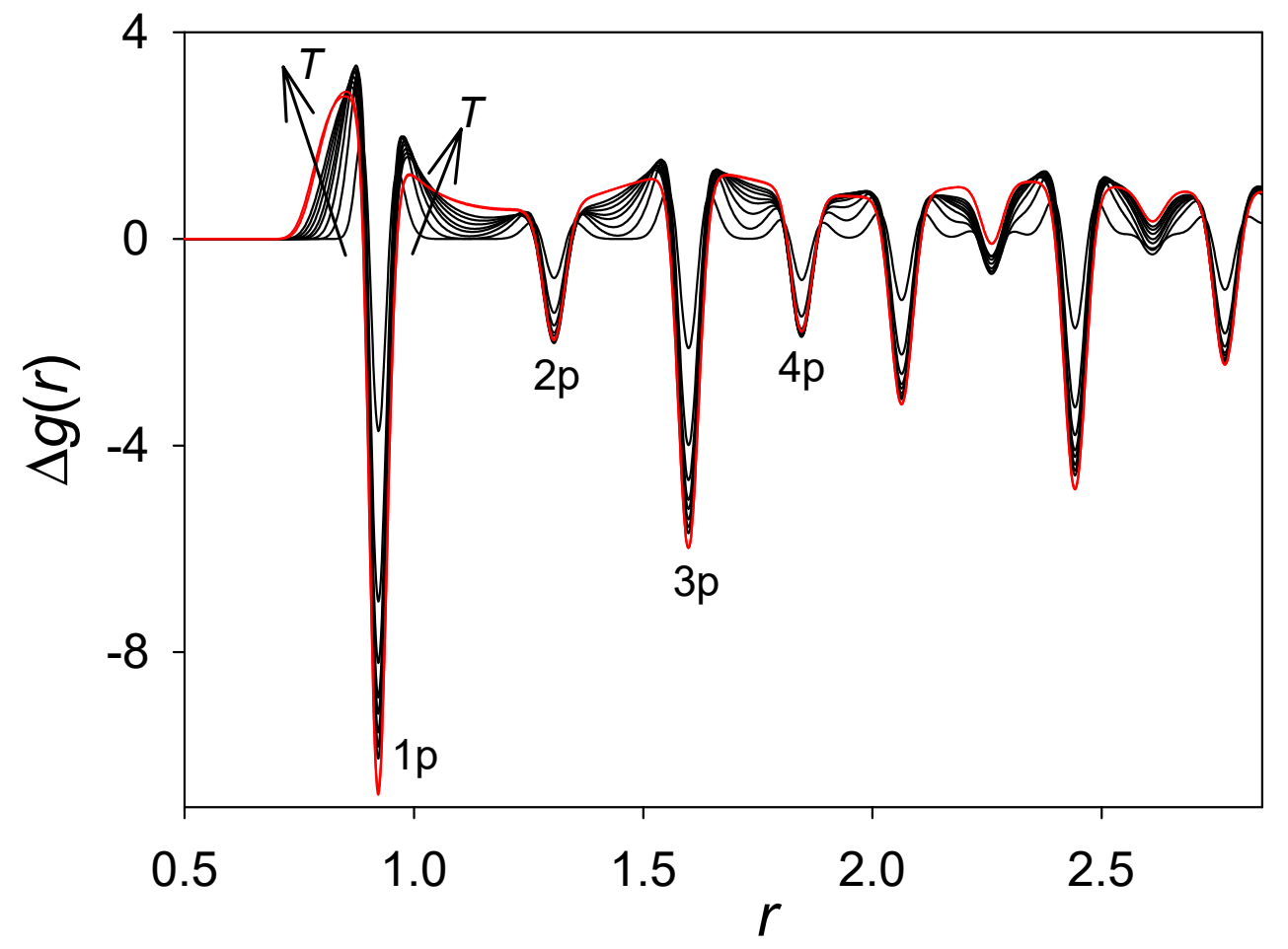

Figure S10: Temperature dependence of $\Delta g(r, T)=g(r, T)-g(r, T=1) .1 \mathrm{p}, 2 \mathrm{p}, \ldots$ mark the positions of the first, second, ... peaks of the PDF, arrows mark increasing temperature. Black curves represent the solid, red curves the fluid phase. 


\section{Low frequency behavior of the vibrational density of states}

Zhang et al. [10] discussed the potential occurrence of a boson peak for a superheated Ni crystal at low frequencies. In their analysis, they normalized the VDOS with the Debye vibrational density of states $\left(\sim \omega^{2}\right)$, which is usually used for investigations of glass-forming liquids [11]. Regardless whether such a peak can really be interpreted as a boson peak, the low frequency behavior of the VDOS is also of interest for the superheated LJ crystal. Therefore, a procedure similar to that of Ref. [10] was applied in the present study. The reduced $\operatorname{VDOS} \rho_{\mathrm{V}}(\omega) / \omega^{2}$ is depicted in Fig. S11 that allows a clear identification of a pronounced peak appearing between $\omega=4.25$ and 5 . This peak is accompanied by smaller oscillations at lower frequencies and larger oscillations at higher frequencies, which, however, are increasingly smeared out as the temperature rises. The peak position shifts to smaller frequencies upon heating. The present results for the LJ fcc-solid correspond to the findings of Zhang et al. [10]. Nevertheless, for an unequivocal postulation of the existence of a boson peak, further examinations are definitely necessary, since a rigorous physical theory describing such a peak is missing. 


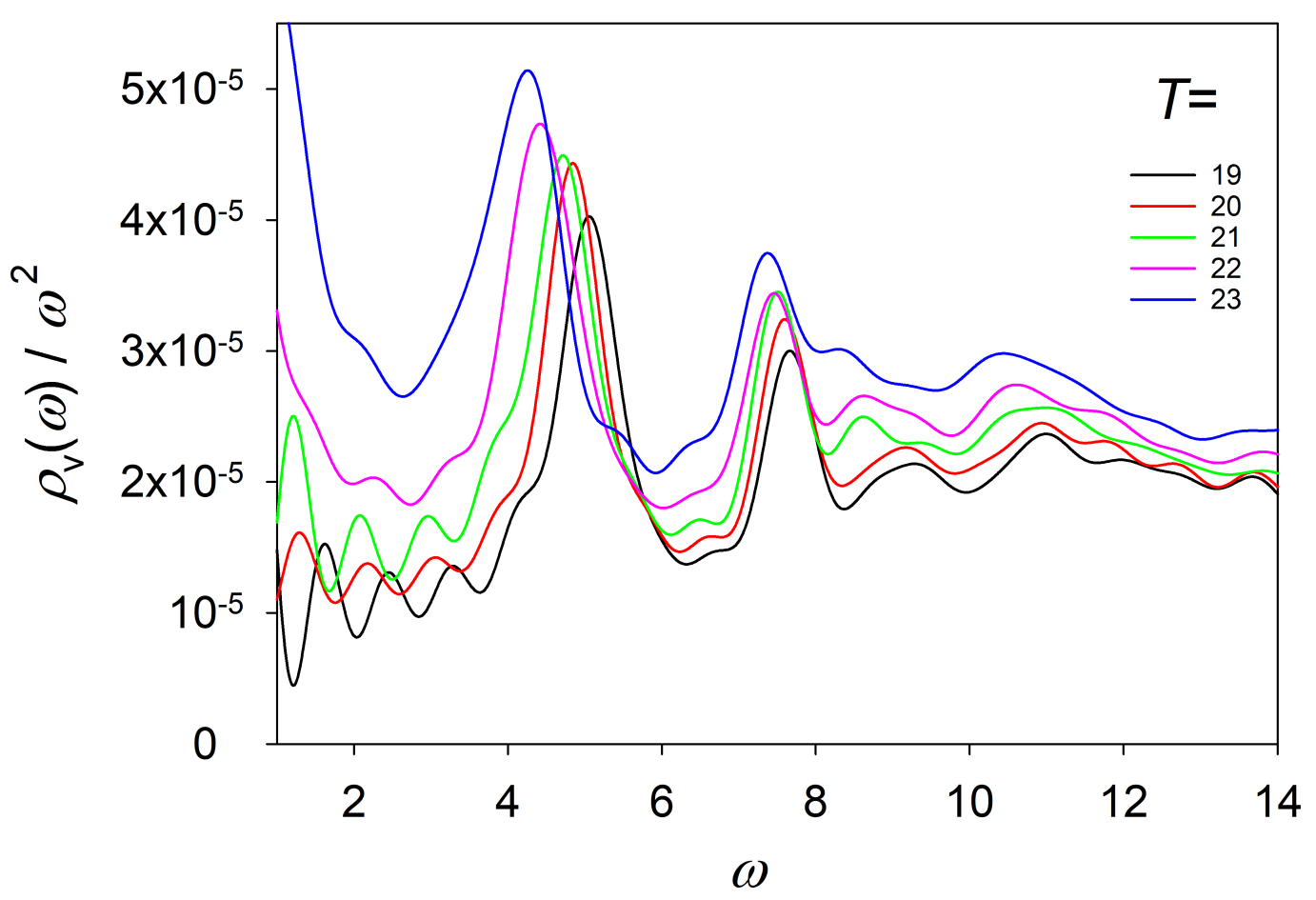

Figure S11: Reduced vibrational density of states $\rho_{\mathrm{V}}(\omega) / \omega^{2}$ along the isochore $\rho=1.8$ for $T=19$ to 23 .

\section{References}

[1] A. Köster, P. Mausbach, and J. Vrabec, "Premelting, solid-fluid equilibria, and thermodynamic properties in the high density region based on the Lennard-Jones potential," J. Chem. Phys. 147, 144502 (2017).

[2] A. J. Schultz and D. A. Kofke, "Comprehensive high-precision high-accuracy equation of state and coexistence properties for classical Lennard-Jones crystals and lowtemperature fluid phases," J. Chem. Phys. 149, 204508 (2018).

[3] R. Lustig, "On the Lennard-Jones and Devonshire theory for solid state thermodynamics," Mol. Phys. 115, 1362 (2017).

[4] A. J. Schultz and D. A. Kofke, Erratum to "Comprehensive high-precision highaccuracy equation of state and coexistence properties for classical Lennard-Jones crystals and low-temperature fluid phases," J. Chem. Phys. 149, 204508 (2018), submitted (2020).

[5] M. Gottschalk, "An EOS for the Lennard-Jones fluid: A virial expansion approach," AIP Adv. 9, 125206 (2019).

[6] A. Ahmed and R. J. Sadus, "Solid-liquid equilibria and triple points of n-6 LennardJones fluids," J. Chem. Phys. 131, 174504 (2009); Erratum 133, 229902 (2010).

[7] J.-P. Hansen and L. Verlet, "Phase Transitions of the Lennard-Jones System," Phys. Rev. 184, 151 (1969).

[8] H. J. Raveché, R. D. Mountain, and W. B. Streett, "Freezing and melting properties of the Lennard-Jones system," J. Chem. Phys. 61, 1970 (1974). 
[9] M. J. Pozo, S. Davis, and J. Peralta, "Statistical distribution of thermal vacancies close to the melting point," Physica B 457, 310 (2015).

[10] H. Zhang, M. Khalkhali, Q. Liu, and J. F. Douglas, "String-like cooperative motion in homogeneous melting," J. Chem. Phys. 138, 12A538 (2013).

[11] T. S. Grigera, V. Martin-Mayor, G. Parisi, and P. Verrocchio, "Phonon interpretation of the 'boson peak' in supercooled liquids," Nature 422, 289 (2003). 